\title{
Imperfect Competition and Market Liquidity with a Supply Informed Trader
}

\author{
Ariadna Dumitrescu*† \\ Universitat Autònoma de Barcelona \\ June, 2003
}

\begin{abstract}
A bstract
We develop a model of insider trading where agents have private information either about liquidation value or about supply and behave strategically to maximize their profits. The supply informed trader plays a dual role in market making and in information revelation. This trader not only reveals a part of the information he owns, but he also induces the other traders to reveal more of their private information. The presence of different types of information decreases market liquidity and induces non-monotonicity of the market indicators with respect to the variance of liquidation value. Replacing the noise introduced by liquidity traders with a random supply also allows us to study the effect the shocks on different components of supply have on prices and quantities.
\end{abstract}

JEL Classification numbers: D82, G12, G14.

Keywords: insider trading, imperfect competition, market liquidity.

\footnotetext{
${ }^{*}$ I am very grateful to Jordi Caballé for his valuable comments and kind guidance. I would like to thank also Amil Dasgupta, Steffen Huck and David Pérez-Castrillo for very helpful comments. All the remaining errors are my own responsability.

${ }^{\dagger}$ Correspondence address: Ariadna Dumitrescu, IDEA, Departament d'Economia i d'Història Econòmica, Universitat Autònoma de Barcelona, Edifici B, Bellaterra (Barcelona), 01893, Spain. Phone: (34) 935811 561. Fax: (34) 935812 012. e-mail: adumit@idea.uab.es
} 


\section{Introduction}

Agents engaged in trading activities might have access to different sources of information: information about fundamentals or information about supply. The existence of different types of information might reduce the inefficiencies that appear when agents are trading on private information about fundamentals. One of the reasons these inefficiencies occur is the fact that market makers respond to the existence of traders with private information by reducing the liquidity of the market. However, it is the private information itself which generates these inefficiencies and not necessarily the market mechanism. In this paper we analyze the process through which different types of information are transmitted to prices and how this affects market performance. We develop a model of insider trading in the context of an imperfectly competitive market where agents have private information either about liquidation value or about supply (different types of information that may influence the security prices at any point in time). In an imperfect competitive equilibrium prices are less informative than in a competitive rational expactations equilibrium. This is due mainly to the fact that a strategic trader exploits his informational advantage taking into account the effect the quantity he chooses is expected to have on both the price and the other informed traders' strategy. This effect is even more important when in the market there exist two types of information. As a result, we study how strategic trading on two types of information affects market liquidity, informational efficiency of prices and other market indicators in this new setup.

We use the framework developed by Kyle $(1985,1989)$ which have become a standard for analyzing strategic noisy rational expectations markets. Kyle's (1985) model explains how a risk neutral informed trader exploits his informational advantage by behaving strategical and shows that the smoothing behaviour of the trader leads to prices that have constant volatility as the time periods become shorter to approach a continuous auction. An important generalization of the Kyle's model is to allow for multiple informed traders. Since the monopolist trader makes positive profits it follows that other trader might be willing to acquire information. Foster and Wiswanathan (1993) and Holden and Subrahmanyam (1992) explore this restriction of a single informed 
trader and point out the contrast between the case of a monopolist and the one of multiple traders. Thus, Foster and Wiswanathan (1993) extend Kyle's model to many traders and a larger class of distributions but obtain that Kyle's result that the informed trader can make positive profits does not hold anymore. On the other hand, Holden and Subrahmanyam (1992) conclude that competition between informed traders leads to fully revelation of information. Kyle (1989), to which our work is closely related, proposes an imperfect competition model in which there are noise traders, price informed traders and uninformed traders. He shows that a strategic trader acts as he trades against a residual supply curve. This implies lower quantities by comparison with the competitive rational expectations equilibrium and, consequently, in equilibrium prices reveal less information than in the competitive case. As it will be emphasized in this paper, in the case we have different types of information the dual role of prices to aggregate information and clear the market is even more important.

In the Kyle-type models an important assumption is the presence of noise. As it was already explained by Grossman and Stiglitz (1980), noise is needed in the model to prevent prices to be fully revealing. They show that in a model in which agents are price takers and prices are fully revealed no agent will be willing to acquire costly information. To overcome this difficulty several ways to introduce noise were used: adding noise traders, considering uncertainty which has a dimension greater than that of price, or assuming that the aggregate endowment is imperfectly observed. We use this last approach by assuming a random supply. The presence of shocks in supply has a significant price impact. A supply shock leads to a change in prices and this determines the investors to revise their expectations. However, if the supply shock is observable by the supply informed traders, these traders make use of their informational advantage and therefore, are willing to adjust their demand. Consequently, we assume that there exists a supply informed trader who receives a signal about supply. This approach was used before by Gennotte and Leland (1990) who consider a model were speculators posses private and diverse information. ${ }^{1}$ They consider price takers

\footnotetext{
${ }^{1} \mathrm{~A}$ similar assumption is that market makers have some information about the uninformed order flow and it can be found in Admati and Pfleiderer (1991) and Madhavan (1992). Palomino (2001) considers also a setup where the informed agents have information both about the liquidation value
} 
speculators who gather information either about prices or about supply and show that these informational differences can cause financial markets to be relatively illiquid. Our model builds on the assumption of Gennotte and Leland (1990) about the existence of a random supply and informed supply speculator but we consider an imperfect competition setup with both price informed and supply informed agents where the agents submit limit orders. In general dealers observe order flow and collect information from multiple sources. Therefore, we can think of the supply informed agent as being a dealer who can observe the order flow. As pointed out by Brown and Zhang (1997), despite of the fact that dealers may be better informed than other traders, in a competitive market they cannot earn rents on the information on the order flow. This is due to the fact that price informed agents use their informational advantage to make gains on the expense of dealers. However, we will see that in our setup of an imperfect competitive market dealers can aggregate the information from trading and use it to earn speculative profits. Thus, the dealers can learn about the liquidation value of the asset from the orders placed by the price informed agents. The information revelation is increased significantly in our setup since the agents are placing limit orders and therefore, they condition their demands on prices and infer in this way a part of others' information. We assume here that there is only one supply informed trader. Made for simplicity, the assumption is in line with the result obtained by Ellis et al. (2001). They show that in general, one dealer tends to dominate the trading on a stock (executing a little more than half of the day's volume). They also answer the question who is the dominant dealer. Depending on the time passed from the offer day, the dominant dealer might be the underwriter, a wholesaler or a generic market maker.

In the rational expectations paradigm traders understand that prices reveal the information they have when they choose the quantities to be traded. The link between information and prices via trades provides an explicit mechanism for information transmission between traders. The existence of private information means that a trader may have incentives to act strategically in order to maximize his profits. Therefore, given his private information, a trader maximizes his conditional expected profits taking into account the effect of his trading on prices and taking as given the strategies other and the quantity traded by one of the noise traders. 
traders use to chose their demand schedules. As in the imperfect competition model of Kyle (1989) we assume further that all the speculators choose strategically the amounts they trade. Therefore, the supply informed speculator will also chose his demand taking into account the effect of his trading on prices and revealing a part of information about the shock in supply to the other market participants. As a result, in our model both the information about the value of the asset or about supply is revealed through the quantities to be traded.

We are interested to understand the effects of different types of information on market liquidity, informativeness of prices, price volatility, and the ability of informed traders to exploit their private information. Our goal is to see how market liquidity and price efficiency are influenced by strategic interaction between agents with different types of private information. Allowing the supply informed agent to behave strategically, has an important role in the market-making and in information revelation. Indeed, he decreases the market depth and increases the amount of information revealed in prices but, unlike in the perfect competitive case, he also makes positive profits. Our model suggests that the presence of different types of information in the market decreases market liquidity. The result is in line with the one of Glosten and Milgrom (1985) that more information in the market leads to an increase in the bidask spread (i.e. a decrease in the market liquidity). The result should be situated in between the one of Kyle $(1985,1989)$ and the subsequent literature which shows that increasing the number of informed traders increases market liquidity, and the one of Subrahmanyam (1991) which shows the opposite. Thus, Subrahmanyam (1991) also obtains that market liquidity can be decreased by increasing the number of informed traders in the case traders are risk averse. In our model we obtain that the presence of the supply informed agent and therefore, of a different type of information in the market, leads to a decrease in market liquidity. Still, if we are increasing the number of price informed traders we will still obtain the increase the market liquidity obtained in Kyle $(1985,1989)$.

We performed comparative statics results for market liquidity measured as market depth and we conclude that if the information received by the supply informed agent is very precise or the one of the price informed agents is very poor the market liquidity 
is low. Most important, the presence of a supply informed agent in the market induces non-monotonicity of the market depth and other market performance indicators. Finally, we study how changes in supply affect the equilibrium price. We will consider two cases: a change in supply known to all investors or a change known only to the supply informed investors. We obtain that price informed agents absorb a higher fraction of the known shock, while the supply informed agent absorbs always half of the unknown shock.

The remainder of this paper is organized as follows. Section 2 presents the model. We establish the information structure and define the imperfect competitive rational equilibrium expectations. Section 3 characterizes the equilibrium. We find an unique linear imperfect competitive rational expectations price function together with agents' demand functions in equilibrium. Section 4 proceeds with the calculation of some market indicators: volatility of prices, informativeness of prices and expected profits. Section 5 contains the characterization the equilibrium in the case there is no supply informed trader and then Section 6 compares the market indicators of this economy with the one of the economy with a supply informed agent. Finally, Section 7 summarizes the results and gives some directions for further research. All the proofs appear in the appendix.

\section{The Model}

The framework is similar to the one in Kyle (1989). However, we assume risk neutrality, absence of uniformed traders and random supply with an observable component for one trader - the supply informed trader. As already pointed out by Kyle (1989), the assumption of existence of uninformed traders does not change the analysis, but their

presence leads to an increase in market depth. In what it follows we make the following assumptions:

A.1 There is a single security in the market that trades at market clearing price $\widetilde{p}$ and yields an exogenous liquidation value $\widetilde{v}$ which has a normal distribution with mean $\bar{v}$ and variance $\sigma_{v}^{2}$. 
A 2 There are $N$ price informed traders, indexed $n=1, \ldots, N$ and a supply informed trader. The price informed trader $n$ observes a private signal $\widetilde{i_{n}}=\widetilde{v}+\widetilde{e_{n}}$. We assume that $e_{n}$ is distributed $N\left(0, \sigma_{e}^{2}\right)$ for all $n=1, \ldots, N$. We suppose that for any $j \neq n \widetilde{e_{j}}$ and $\widetilde{e_{n}}$ are uncorrelated and moreover, they are uncorrelated with all the other random variables in the model. The supply informed trader observes a private signal $S$ which is normal distributed with mean 0 and variance $\sigma_{S}^{2}>0$.

A.3 The random supply that keeps the traders from perfectly inferring the aggregate information from prices is modelled in a similar manner to the one in Gennotte and Leland (1990). The net supply $\widetilde{m}$ consists of a fixed amount $\bar{m}$ and a random supply $\widetilde{S}$ distributed $N\left(0, \sigma_{S}^{2}\right)$. This liquidity shock $\widetilde{S}$ is observed only by the supply informed trader.

A.4 Agents are risk neutral and behave strategically taking into account the effect of their trading on prices.

As in Kyle (1989), the $n^{\text {th }}$ price informed trader has a strategy $X_{n}$ which is a mapping from $R^{2}$ (the cartesian product of the set of asset prices and the set of his signals) to $\mathrm{R}$ (the set of shares he desires to trade), $X_{n}(\cdot, \cdot): \mathrm{R}^{2} \longrightarrow \mathrm{R}$. After observing his signal $i_{n}$, each price informed trader submits a demand schedule (or generalized limit order) $X_{n}\left(\cdot, \widetilde{i}_{n}\right)$, which depends upon his signal. Similarly, the supply informed trader has a strategy $Y$ which is a mapping from $\mathbf{R}^{2}$ (the cartesian product of the set of asset prices and the set of his signals) to $R$ (the set of shares he wants to trade), $Y(\cdot, \cdot): \mathbf{R}^{2} \longrightarrow \mathrm{R}$. After observing the signal $S$, the supply informed trader chooses a demand schedule $Y(\cdot, S)$, which depends upon that signal. Notice that since $\bar{m}$ is known by everyone, this implies that the supply informed agent actually knows $\widetilde{m}$. Given a market clearing price $p$, the quantities traded by price informed traders and supply informed trader can be written $x_{n}=X_{n}\left(p, i_{n}\right), n=1, \ldots, N$ and $y=Y(p, S)$. In the above notations a tilde distinguishes a random variable from its realization. Thus, $x_{n}$ denotes a particular realization of $\widetilde{x}_{n}$. The assumption that the price informed and the supply informed agents submit limit orders for execution against existing limit orders submitted by the other market participants turns out to be very important (for a detailed discussion see Kyle (1989)). In this context both the price informed and the 
supply informed agents provide liquidity and therefore, have a market making role in the market.

The price of the asset is set such that the market clears. The traders submit their demand schedules to an auctioneer who aggregates all the schedules submitted, calculates the market clearing price and allocates quantities to satisfy traders' demand. Thus, the market clearing price $\widetilde{p}$ should satisfy with probability one

$$
\sum_{n=1}^{N} X_{n}\left(\widetilde{p}, \widetilde{i}_{n}\right)+Y(\widetilde{p}, \widetilde{S})=\widetilde{m}
$$

To emphasize the dependence of the market-clearing price on the strategies of the traders we write

$$
p=p(X, Y), x_{n}=x_{n}(X, Y), y=y(X, Y)
$$

where $X$ is the vector of strategies of price informed traders defined by $X=\left(X_{1}, \ldots, X_{N}\right)$ and $Y$ is the strategy of the supply informed trader.

The traders are risk neutral and maximize expected profits. The profits of the price informed trader $n$ and supply informed trader are, respectively, given by

$$
\widetilde{\pi}_{n}^{P I}=(\widetilde{v}-\widetilde{p}(X, Y)) \widetilde{x}_{n}(X, Y), \quad \widetilde{\pi}^{S I}=(\widetilde{v}-\widetilde{p}(X, Y)) \widetilde{y}(X, Y)
$$

With these notations, following Kyle (1989) we can proceed to define a rational expectations equilibrium in our setup.

Definition 1 An imperfectly competitive rational expectations equilibrium is defined as a vector $(X, Y, p)$, where $X$ is a vector of strategies of the price informed agents $X=\left(X_{1}, \ldots, X_{N}\right), Y$ is a strategy of the supply informed agent and $p$ is the equilibrium price such that the following conditions hold:

1. For all $n=1, \ldots, N$ and for any alternative strategy vector $X^{\prime}$ differing from $X$ only in the $n^{\text {th }}$ component $X_{n}$, the strategy $X$ yields a higher profit than $X^{\prime}$ :

$$
\begin{aligned}
& E_{n}\left[(\widetilde{v}-\widetilde{p}(X, Y)) \widetilde{x}_{n}(X, Y) \mid \widetilde{p}(X, Y)=p, \widetilde{i}_{n}=i\right] \geq \\
& E_{n}\left[\left(\widetilde{v}-\widetilde{p}\left(X^{\prime}, Y\right)\right) \widetilde{x}_{n}\left(X^{\prime}, Y\right) \mid \widetilde{p}\left(X^{\prime}, Y\right)=p, \widetilde{i}_{n}=i\right]
\end{aligned}
$$


2. For any alternative strategy $Y^{\prime}$ the strategy $Y$ yields a higher profit than $Y^{\prime}$ :

$$
\begin{aligned}
& E[(\widetilde{v}-\widetilde{p}(X, Y)) \widetilde{y}(X, Y) \mid \widetilde{p}(X, Y)=p, \widetilde{S}=S] \geq \\
& E\left[\left(\widetilde{v}-\widetilde{p}\left(X^{\prime}, Y\right)\right) \widetilde{y}\left(X, Y^{\prime}\right) \mid \widetilde{p}\left(X, Y^{\prime}\right)=p, \widetilde{S}=S\right] .
\end{aligned}
$$

3. The price $p=\widetilde{p}(X, Y)$ clears the market (with probability one) i.e.

$$
\sum_{n=1}^{N} X_{n}\left(\widetilde{p}, \widetilde{i}_{n}\right)+Y(\widetilde{p}, \widetilde{S})=\widetilde{m}
$$

This defines a Nash equilibrium in demand functions. Given their private information, traders maximize their conditional expected profits taking into account the effect

of their trading on prices and taking as given the strategies other traders use to choose their demand schedules.

We look for a symmetric linear Bayesian Nash Equilibrium as in Kyle (1989), that is, an equilibrium where the strategies $X_{n}$ and $Y$ are linear functions:

$$
\begin{aligned}
X_{n}\left(\widetilde{p}, \widetilde{i_{n}}\right) & =\alpha^{P I}+\beta^{P I} \widetilde{i}_{n}-\gamma^{P I} \widetilde{p}, \text { for any } n=1, \ldots, N \text { and } \\
Y(\widetilde{p}, \widetilde{S}) & =\alpha^{S I}+\beta^{S I} \widetilde{S}-\gamma^{S I} \widetilde{p},
\end{aligned}
$$

where $\alpha^{P I}, \beta^{P I}, \gamma^{P I}, \alpha^{S I}, \beta^{S I}, \gamma^{S I} \in \mathrm{R}$.

With this assumption we can infer from the market clearing condition that the equilibrium price is given by

$$
p=\left(N \gamma^{P I}+\gamma^{S I}\right)^{-1}\left(N \alpha^{P I}+\alpha^{S I}+\beta^{P I} \sum_{n=1} \widetilde{i}_{n}+\beta^{S I} \widetilde{S}-\widetilde{m}\right) .
$$

\section{Characterization of the Equilibrium}

We describe in the following proposition the equations that characterize the symmetric Bayesian-Nash equilibrium. This equilibrium has linear trading rules and linear pricing rule and is shown to be unique among all linear, symmetric Bayesian-Nash equilibria. As in most Kyle type models, the linearities are not ex-ante imposed in the agents strategy sets: as long as the informed traders use a linear trading strategy, the market maker will use a linear pricing rule and vice versa. 
Proposition 1 If $N(N-2) \geq \frac{\sigma_{e}^{2}}{\sigma_{v}^{2}}$ there exists a unique linear symmetric equilibrium defined as:

$$
\begin{aligned}
X_{n}\left(\widetilde{p}, \widetilde{i}_{n}\right) & =\alpha^{P I}+\beta^{P I} \widetilde{i}_{n}-\gamma^{P I} \widetilde{p}, \text { for any } n=1, \ldots, N \text { and } \\
Y(\widetilde{p}, \widetilde{S}) & =\alpha^{S I}+\beta^{S I} \widetilde{S}-\gamma^{S I} \widetilde{p}
\end{aligned}
$$

with $\quad \alpha^{P I}, \beta^{P I}, \gamma^{P I}, \alpha^{S I}, \beta^{S I}, \gamma^{S I}$ given by

$$
\begin{aligned}
\alpha^{P I} & =\frac{\sigma_{e}^{2}\left(N(3 N-2) \sigma_{v}^{2}+(2 N-1) \sigma_{e}^{2}\right) \delta^{1 / 2}}{2 N^{2} \sigma_{v}\left(N^{2} \sigma_{v}^{2}+\sigma_{e}^{2}\right)\left(N \sigma_{v}^{2}+\sigma_{e}^{2}\right)} \bar{v}+\frac{N(N-2) \sigma_{v}^{2}-\sigma_{e}^{2}}{N\left(N^{2} \sigma_{v}^{2}+\sigma_{e}^{2}\right)} \bar{m} \\
\beta^{P I} & =\frac{\delta^{1 / 2}}{2 N\left(N \sigma_{v}^{2}+\sigma_{e}^{2}\right)} \\
\gamma^{P I} & =\frac{\left(N^{2} \sigma_{v}+(2 N-1) \sigma_{e}^{2}\right) \delta^{1 / 2}}{2 N^{2} \sigma_{v}^{2}\left(N^{2} \sigma_{v}^{2}+\sigma_{e}^{2}\right)} \\
\alpha^{S I} & =-\frac{(N-1)\left(N^{2} \sigma_{v}^{2}+(2 N-1) \sigma_{e}^{2}\right) \sigma_{e}^{2} \delta^{1 / 2}}{2 N^{2} \sigma_{v}^{2}\left(N \sigma_{v}^{2}+\sigma_{e}^{2}\right)\left(N^{2} \sigma_{v}^{2}+\sigma_{e}^{2}\right)} \bar{v}+\frac{N^{2} \sigma_{v}^{2}+(2 N-1) \sigma_{e}^{2}}{N\left(N^{2} \sigma_{v}^{2}+\sigma_{e}^{2}\right)} \bar{m} \\
\beta^{S I} & =\frac{N^{2} \sigma_{v}^{2}+(2 N-1) \sigma_{e}^{2}}{2 N\left(N \sigma_{v}^{2}+\sigma_{e}^{2}\right)} \\
\gamma^{S I} & =-\frac{(N-1) \sigma_{e}^{2}\left(N^{2} \sigma_{v}^{2}+(2 N-1) \sigma_{e}^{2}\right) \delta^{1 / 2}}{2 N^{2} \sigma_{v}^{2}\left(N \sigma_{v}^{2}+\sigma_{e}^{2}\right)\left(N^{2} \sigma_{v}^{2}+\sigma_{e}^{2}\right)}
\end{aligned}
$$

where

$$
\delta \equiv \frac{\left(N(N-2) \sigma_{v}^{2}-\sigma_{e}^{2}\right)\left(N^{2} \sigma_{v}^{2}+\sigma_{e}^{2}\right) \sigma_{S}^{2}}{(N-1) \sigma_{e}^{2}}
$$

The condition $N(N-2) \geq \frac{\sigma_{e}^{2}}{\sigma_{v}^{2}}$ is similar to the usual condition $N>2$ in all Kyletype models. It tells us that we need competition in order to alleviate the asymmetric information problem. In our model the asymmetric information problem is even more important than in Kyle $(1985,1989)$ because we have two different types of information that aggregates in prices. Since the supply informed agent observes the supply he acts as an informational monopolist trading such that he always extracts some rents. However, the price informed agents are competing against him trying to reduce his informational advantage. The worse the quality of the signal of the price informed traders relative to the liquidation value, the more difficult is for them to compete against 
the supply informed. However, since they are asymmetrically informed, increasing their number it will make it more difficult for the supply informed to infer their information. Consequently, in the case we have a supply informed agent we need more competition in order to reduce his monopoly power and trade aggressiveness and therefore, for the equilibrium to exists.

We would like to understand the effects of different types of information on market liquidity, informativeness of prices, price volatility, and the ability of informed traders to exploit their private information. We are first concerned with market liquidity because it has been recognized as an important determinant of market behaviour. There are different measures of market liquidity used in the literature: market depth, bid-ask spread and price movement after trade. We will use as a measure of liquidity the market depth (as defined by Kyle (1989)), which represents the volume of trading needed to move prices one unit. While solving the above system we have obtained that

$$
\gamma=N \gamma^{P I}+\gamma^{S I}=\frac{\left(N^{2} \sigma_{v}^{2}+(2 N-1) \sigma_{e}^{2}\right) \delta^{1 / 2}}{2 N^{2} \sigma_{v}^{2}\left(N \sigma_{v}^{2}+\sigma_{e}^{2}\right)} .
$$

On the other hand, from the price equation (3) we can see that an increase (decrease) in the supply by $\gamma$ induces the price to fall (rise) by one dollar. The trading volume needed to move the price by one unit (market depth) was used by Kyle (1985) as a measure of market liquidity. We use the same measure as Kyle and consequently, $\gamma$ is our measure of the market liquidity. As we can see the market depth $\gamma$ has two components that have opposite effect. The first component $N \gamma^{P I}$ is attributed to the price informed agents trading. This is the amount with which they contribute to a change in the price when each of them trades an additional unit. The more priced informed agents are in the market, the higher the liquidity. Similarly, we have that $\gamma^{S I}$ is the change in price due to an additional unit of trading by the supply informed agent. The two components have opposite sign and we have here a trade-off: whenever the price informed agents are increasing the market liquidity the supply informed agent will try to reduce it.

The fact that $\gamma^{S I}$ is negative is a very important result in our model and it is a consequence of the mechanism of information transmission through prices. In general, with asymmetric information prices play a dual role of information aggregation and 
market clearing. The role of information aggregation played by prices is even more important in our economy with asymmetric and different information. We have thus, two important channels through which we have flow of information (information about the liquidation value from the price informed traders towards the supply informed trader and information about supply from the supply informed trader towards the price informed traders). The supply informed agent puts a positive weight on price $\left(\gamma^{S I}<0\right)$ because when he sees an increase in price he associates it with good news about the liquidation value (he knows the supply, so the price increase cannot be due to a decrease in supply). This mechanism of information transmission actually triggers a decrease in market liquidity. For 1 additional unit demanded by a price informed agent the price goes up. The supply informed agent associates it with good news about the liquidation value and increases his demand leading to a even higher increase in price. Since the same volume will increase the price more we can conclude that we have a decrease in the market liquidity.

Next, let us investigate how the market depth varies with the parameters of the model: the variance of the liquidity shock $\sigma_{S}^{2}$, the variance of signals $\sigma_{e}^{2}$, and the variance of the liquidation value $\sigma_{v}^{2}$.

Corollary 1 (i) Market depth is increasing in the variance of liquidity shock $\widetilde{S}, \sigma_{S}^{2}$.

(ii) Market depth is decreasing in the variance of the error of the signal received by price informed agents $\sigma_{e}^{2}$.

(iii) Market depth viewed as a function of the variance of liquidation value $\sigma_{v}^{2}$ has an inverted U-shaped.

(iv) Market depth is decreasing in the relative quality of the signals $\frac{\sigma_{e}^{2}}{\sigma_{v}^{2}}$.

As we have seen before, the market depth has two components $\gamma=N \gamma^{P I}+\gamma^{S I}$. The first component is the contribution to the market depth of trades executed by price informed agents while the second one is the contribution to the market depth of trades executed by the supply informed agent. The two components have opposite effect and thus, the final result on market depth due to the market making activity of the agents depends on which of the two components dominates. The first result in the Corollary is similar to the previous ones in the literature (Kyle (1985) and the 
other imperfect competition models). It tells us that the higher the variance of the supply (in the other papers -the variance of the noise trading), the easier is for the price informed agents to hide and therefore, to make use of their informational advantage (the volume needed to move the price is higher, and this helps them to trade better on their information without being discovered). If the signal of the supply informed agent is very informative he reduces the market liquidity. Otherwise, he might infer wrongly the information embedded in prices and therefore, contribute himself to the increase in the market liquidity. The second result claims that if the signal of the price informed agents is very precise, the market depth is high. This happens because when the price informed agents have poor informational advantage, they trade less aggressively and devote more to market making activities. Notice that these results indicate that the effect on market depth of the trades of price informed agents dominates the effect of the trades of the supply informed agent for all values of $\sigma_{S}^{2}$ or $\sigma_{e}^{2}$. The third result is somehow different from the previous results. This difference is triggered exactly by the existence of a supply informed agent. Here we have that the effect on market depth of the trades of supply informed agent may dominate the effect of the trades of the price informed agents when the variance of liquidation value is high. If the variance $\sigma_{v}^{2}$ is small, the signal the price informed receive is better and the supply informed agent is not able to decrease the market liquidity. However, as the variance of liquidation value $\sigma_{v}^{2}$ increases, we have more competition in the market and therefore a decrease in the market depth. Finally, we see that the effect of changing $\sigma_{e}^{2}$ always dominates the one of $\sigma_{v}^{2}$, the market liquidity being always decreasing in $\frac{\sigma_{e}^{2}}{\sigma_{v}^{2}}$.

We do obtain in our model that the behaviour of the market depth with respect to the variance of the supply and the variance of the error of the signal is very similar to the previous cases in the literature, but overall the quantitative result it is very different. We obtain that the presence of a supply informed decreases the market liquidity. Our result should be interpreted as it follows: if we have different types of information in the market, the liquidity is reduced. The result should be situated in between the one of Kyle $(1985,1989)$ and the subsequent literature which show that increasing the number of informed traders increases market liquidity, and the one of Subrahmanyam (1991) which shows the opposite. Thus, Subrahmanyam (1991) also 
obtains that market liquidity can be decreased by increasing the number of informed traders in the case the market maker is risk averse. In our model we obtain that the presence of the supply informed agent and therefore, of a different type of information, leads to a decrease in market liquidity. This result captures the intuition of Glosten and Milgrom (1985), that more information in the market decreases the market liquidity. In their model, they use as a measure of liquidity the bid-ask spread (low liquidity being equivalent to high bid-ask spread), and an increase in the number of informed agents increases the bid-ask spread. Still, if we are increasing the number of price informed traders we will have again the increase the market liquidity obtained in Kyle (1985, 1989). Despite of the fact that the decrease in the market liquidity is due to the different type of information, our result is very similar to the one of Subrahmanyam (1991). The similitude is caused by the fact that the supply informed agent is risk neutral, but he behaves strategically. Moreover, since he submits limit orders he has a market-making role, the role played by him in the economy being thus similar to the one played by the risk-averse market maker in Subrahmanyam's (1991) model.

Once we have determined the equilibrium demand strategies we can determine also the market clearing price.

Corollary 2 The equilibrium price is given by

$$
\begin{aligned}
\widetilde{p} & =\frac{\sigma_{e}^{2}(2 N-1)}{N^{2} \sigma_{v}^{2}+(2 N-1) \sigma_{e}^{2}} \bar{v}+\frac{N \sigma_{v}^{2}}{N^{2} \sigma_{v}^{2}+(2 N-1) \sigma_{e}^{2}} \sum_{n=1}^{N} \widetilde{i}_{n} \\
& -\frac{N \sigma_{v}^{2}\left(N^{2} \sigma_{v}^{2}+\sigma_{e}^{2}\right)}{\left(N^{2} \sigma_{v}^{2}+(2 N-1) \sigma_{e}^{2}\right) \delta^{1 / 2}} \widetilde{S}-\frac{2 N \sigma_{v}^{2}\left(N \sigma_{v}^{2}+\sigma_{e}^{2}\right)}{\left(N^{2} \sigma_{v}^{2}+(2 N-1) \sigma_{e}^{2}\right) \delta^{1 / 2}} \bar{m}
\end{aligned}
$$

From this corollary we can see that the unconditional expectation of the equilibrium price is

$$
E(\widetilde{p})=\bar{v}-\frac{2 N \sigma_{v}^{2}\left(N \sigma_{v}^{2}+\sigma_{e}^{2}\right)}{\left(N^{2} \sigma_{v}^{2}+(2 N-1) \sigma_{e}^{2}\right) \delta^{1 / 2}} \bar{m}
$$

and it depends on the expected supply $\bar{m}$. If $\bar{m}=0$, the price is an unbiased estimator of $\bar{v}$, but it is biased if $\bar{m} \neq 0$. We also can see that as expected the higher the supply (the expected supply $\bar{m}$, or the realization of the liquidity shock $\widetilde{S}$ observed by the supply informed agent), the lower the price and the higher the signals received by the price informed agents the higher the price. 
Notice also that a change in the different components of the supply has a different impact on price. A change in the known part of supply $\bar{m}$ is absorbed by the agents through the quantity demanded in a proportion of $\frac{N-1}{N}$ (we have seen while calculating the strategies that $\alpha=N \alpha^{P I}+\alpha^{S I}=g\left(N, \sigma_{v}^{2}, \sigma_{e}^{2}\right)+\frac{(N-1)}{N} \bar{m}$, where $g\left(N, \sigma_{v}^{2}, \sigma_{e}^{2}\right)$ is the function we had obtained in the Appendix) and only $\frac{1}{N}$ is reflected in price. Similarly, a shock in the component of supply known to supply informed agent $\widetilde{S}$ is absorbed half by this agent through his demand and partly is reflected in price. As I have already explained, the supply informed trader has a monopolist position and extracts rents that amount, as we saw above, to half of the unknown component of supply.

\section{Market Indicators}

In what it follows we study the implications the existence of a supply informed agent have on the market performance. We compute some market indicators: volatility of prices, informativeness of prices and expected profits of different market participants and characterize them with respect to the variance of the liquidation value of the asset.

Corollary 3 The price volatility, measured as the variance of price, is

$\operatorname{Var}(\widetilde{p})=\frac{N^{2}(N-2)\left(\sigma_{v}^{2}\right)^{2}+N \sigma_{v}^{2} \sigma_{e}^{2}\left(2 N^{2}-3 N-1\right)-\left(\sigma_{e}^{2}\right)^{2}}{\left(N(N-2) \sigma_{v}^{2}-\sigma_{e}^{2}\right)}\left(\frac{N \sigma_{v}^{2}}{N^{2} \sigma_{v}^{2}+(2 N-1) \sigma_{e}^{2}}\right)^{2}$

Similar to the case when there exists no supply informed agent we have that the volatility of prices does not depend on the noise in supply. If the noise in supply increases all the agents - both the price informed and the supply informed - trade more aggressively making better use of their particular informational advantage. We can also see that price volatility may decrease or increase with the variance of the liquidation value $\sigma_{v}^{2}$. We obtain thus that the price volatility has a $\mathrm{U}$ shape as respect to $\sigma_{v}^{2}$. When the variance of the liquidation value $\sigma_{v}^{2}$ is small there is not too much information revealed. But as we have seen if $\sigma_{v}^{2}$ increases, the market depth decreases and this leads to more information revelation. Consequently, when $\sigma_{v}^{2}$ increases the prices become 
more volatile just because they contain more information. It is interesting to notice that if the competition increases the range in which the volatility of prices is a decreasing function of $\sigma_{v}^{2}$ shrinks and we recover the result from the case without supply informed trader that the higher the variance of the liquidation value of the asset, the higher the volatility of prices. As a result, in a market where there are enough price informed agents, there is more information revelation and the volatility of prices increases.

Next, we would like to find which is the amount of private information - both about the liquidation value and supply - that is revealed through prices. We define thus, the information content of prices as the difference between the prior variance of the payoff and the variance conditional on prices. Using the normality assumptions we obtain the expression presented in the following Corollary:

Corollary 4 The information content of prices is

$$
\operatorname{Var}(\widetilde{v})-\operatorname{Var}(\widetilde{v} \mid \widetilde{p})=\frac{N \sigma_{v}^{2}\left(N(N-2) \sigma_{v}^{2}-\sigma_{e}^{2}\right)}{N^{2}(N-2)\left(\sigma_{v}^{2}\right)^{2}+N \sigma_{v}^{2} \sigma_{e}^{2}\left(2 N^{2}-3 N-1\right)-\left(\sigma_{e}^{2}\right)^{2}} .
$$

Similarly to the previous Corollary, we obtain here also that price efficiency or the information content of prices does not depend on the variance of supply shock $\widetilde{S}$. Moreover, we obtain that informativenes of prices is increasing the variance of the liquidation value $\sigma_{v}^{2}$ and decreasing in the variance $\sigma_{e}^{2}$. These results tells us that when it is difficult to predict the liquidation value or when the signals of price informed agents are poor, the prices play a very important role in information revelation. While these results, are qualitatively similar to the case without supply informed agent, as we will see later they are quantitatively different.

Let us turn to the expected volume traded by the price informed agent and supply informed agent, respectively.

Corollary 5 The expected volume traded by a price informed agent is

$E\left(\left|x_{n}\right|\right)=\frac{2(N-1) \sigma_{v}^{2} \bar{m}}{N^{2} \sigma_{v}^{2}+\sigma_{e}^{2}}+\frac{\left(\frac{2}{\pi}\right)^{1 / 2}}{4 N^{2}}\left(\frac{\left(N^{2} \sigma_{v}^{2}+\sigma_{e}^{2}\right)^{2}+N\left(N \sigma_{v}^{2}+\sigma_{e}^{2}\right)^{2}}{\left(N \sigma_{v}^{2}+\sigma_{e}^{2}\right)^{2}\left(N^{2} \sigma_{v}^{2}+\sigma_{e}^{2}\right)^{2}}\left(\sigma_{v}^{2}+\sigma_{e}^{2}\right) \delta+\sigma_{S}^{2}\right)$. 
The expected volume traded by the supply informed trader is

$E(|y|)=\frac{2\left(N \sigma_{v}^{2}+\sigma_{e}^{2}\right) \bar{m}}{\left(N^{2} \sigma_{v}^{2}+\sigma_{e}^{2}\right)}+\left(\frac{1}{8 \pi}\right)^{1 / 2} \sigma_{S}^{2}\left(1+\frac{(N-1) \sigma_{e}^{2}\left(N(N-2) \sigma_{v}^{2}-\sigma_{e}^{2}\right)\left(\sigma_{v}^{2}+\sigma_{e}^{2}\right)}{N\left(N^{2} \sigma_{v}^{2}+\sigma_{e}^{2}\right)\left(N \sigma_{v}^{2}+\sigma_{e}^{2}\right)^{2}}\right)$.

The expected volume traded by price informed agents and supply informed agent depend positively on the expected supply $\bar{m}$ and the variance of the supply shock $\sigma_{S}^{2}$. However, both the effects of an increase in $\sigma_{S}^{2}$ and in $\bar{m}$ are stronger in the case of supply informed trader. This is the role we wanted actually the supply informed agent to have. Since he has information about supply he captures a big part of the shocks. Finally, the comparative statics with respect to the variance of the liquidation value $\sigma_{v}^{2}$ and the one of the error $\sigma_{e}^{2}$ are ambiguous. In the case without supply informed we have that the expected volume traded by the informed agents increases when the the variance of liquidation value $\sigma_{v}^{2}$ increases and the variance the error $\sigma_{e}^{2}$ decreases. Actually, when the ratio of the variance of the error to the variance of the liquidation value $\sigma_{v}^{2}$ increases (so the quality of his signal decreases) the expected volume traded increases because the agent has not good informational advantage. However, the presence of a supply informed agent diminishes the informational advantage of the price informed agents and therefore, they are forced to trade more aggresively on their information.

We compute next the unconditional profits for all agents.

Corollary 6 The unconditional expected profit of the $n^{\text {th }}$ price informed agent is

$$
\begin{aligned}
\Pi_{n}^{P I}= & E\left(\widetilde{\pi}_{n}^{P I}\right)=\frac{\sigma_{v}^{2} \delta^{1 / 2}(N-1) \sigma_{e}^{2}}{2 N\left(N^{2} \sigma_{v}^{2}+(2 N-1) \sigma_{e}^{2}\right)\left(N \sigma_{v}^{2}+\sigma_{e}^{2}\right)}\left(\frac{N\left(N \sigma_{v}^{2}+\sigma_{e}^{2}\right)}{\left(N(N-2) \sigma_{v}^{2}-\sigma_{e}^{2}\right)}-\right. \\
& \left.\frac{(N-1) \sigma_{e}^{2}}{\left(N^{2} \sigma_{v}^{2}+\sigma_{e}^{2}\right)}\right)+\frac{(N-1)}{\left(N^{2} \sigma_{v}^{2}+\sigma_{e}^{2}\right)} \frac{2 N \sigma_{v}^{2}\left(N \sigma_{v}^{2}+\sigma_{e}^{2}\right)}{\left(N^{2} \sigma_{v}^{2}+(2 N-1) \sigma_{e}^{2}\right) \delta^{1 / 2}} \bar{m}^{2} .
\end{aligned}
$$

The unconditional profit of the supply informed agent is

$$
\begin{aligned}
\Pi^{S I}= & E\left(\widetilde{\pi}^{S I}\right)=\frac{\delta^{1 / 2}(N-1) \sigma_{e}^{2} \sigma_{v}^{2}}{2\left(N^{2} \sigma_{v}^{2}+(2 N-1) \sigma_{e}^{2}\right)}\left(\frac{(N-1) \sigma_{e}^{2}}{\left(N^{2} \sigma_{v}^{2}+\sigma_{e}^{2}\right)\left(N \sigma_{v}^{2}+\sigma_{e}^{2}\right)}+\right. \\
& \left.\frac{N}{\left(N(N-2) \sigma_{v}^{2}-\sigma_{e}^{2}\right)}\right)+\frac{2 N \sigma_{v}^{2}\left(N \sigma_{v}^{2}+\sigma_{e}^{2}\right)}{\left(N^{2} \sigma_{v}^{2}+(2 N-1) \sigma_{e}^{2}\right) \delta^{1 / 2}} \frac{\left(N \sigma_{v}^{2}+\sigma_{e}^{2}\right)}{\left(N^{2} \sigma_{v}^{2}+\sigma_{e}^{2}\right)} \bar{m}^{2} .
\end{aligned}
$$

As we expected, allowing the supply informed agent to behave strategically allows him to make positive profits by comparison with the case of perfect competition when 
he is making zero profits. Notice also that since the price informed traders absorb always $\frac{1}{2 N}$ of the shock $S$, it is actually the different information that they receive the one that makes them have different profits. We want to see also which is the impact of changes in supply on the equilibrium price and the quantity demanded by the different agents. Similar to Gennotte and Leland (1990) we study the two following cases: a supply increase known to all agents $\bar{m}$, and a supply increase known only to supply informed agent $\widetilde{S}$.

Corollary 7 A positive shock in supply known to all the agents $\bar{m}$ leads to an increase in the demand of both type of agents, a decrease in the equilibrium price and therefore, to an increase in the expected profits of both type of agents.

As expected, an increase in the supply known to all agents determines them to adjust their demands according with the existent supply, and it also leads to a decrease of the equilibrium price. We obtain here that the price informed agents are always absorbing a greater proportion of the shock in supply $\bar{m}$.

Corollary 8 A positive shock in the component of supply $\widetilde{S}$, known to the supply informed agent decreases the equilibrium price and increases the quantities demanded by both price informed and supply informed agents.

As expected, in the case of a positive shock in the supply $\widetilde{S}$, the supply informed agent increases his demand making use of the private information he has. Moreover, the increase in supply (due to a positive shock in $\widetilde{S}$ ) absorbed by the supply informed agent is $N$ times higher than the increase of supply absorbed by any price informed agents. An interesting result is that the supply informed agent is always absorbing half of the unobservable shock in supply, the other half being absorbed by the price informed agents.

\section{Equilibrium without Supply Informed A gent}

In order to see which are the effects of different types of information on market liquidity, informativeness of prices, price volatility, and the ability of informed traders to exploit 
their private information we need to provide a benchmark for making comparison with the equilibrium characterized in the previous section. A first step will be to see how the presence in the market of a supply informed agent affects all these market structure indicators. For that we characterize first, in a similar manner, the equilibrium without a supply informed agent. Notice that this model is a version of Kyle's (1989) model with the difference that we do not have uniformed agents and we replace the noise agents by a random supply.

Proposition 2 There exists a unique linear symmetric equilibrium defined as:

$$
X_{I, n}\left(\widetilde{p}, \widetilde{i}_{n}\right)=\alpha_{I}+\beta_{I} \widetilde{i}_{n}-\gamma_{I} \widetilde{p}, \text { for any } n=1, \ldots, N
$$

where $\alpha_{I}, \beta_{I}, \gamma_{I}$ are given by

$$
\begin{aligned}
\alpha_{I} & =\frac{2 \sigma_{e}^{2}}{N \sigma_{v}^{2}}\left(\frac{(N-2) \sigma_{S}^{2}}{N(N-1) \sigma_{e}^{2}}\right)^{1 / 2} \bar{v}+\frac{(N-2)}{N(N-1)} \bar{m} \\
\beta_{I} & =\left(\frac{(N-2) \sigma_{S}^{2}}{N(N-1) \sigma_{e}^{2}}\right)^{1 / 2} \\
\gamma_{I} & =\frac{N \sigma_{v}^{2}+2 \sigma_{e}^{2}}{N \sigma_{v}^{2}}\left(\frac{(N-2) \sigma_{S}^{2}}{N(N-1) \sigma_{e}^{2}}\right)^{1 / 2} .
\end{aligned}
$$

Similarly to the case with supply informed agent we proceed with the calculations of the equilibrium price and equilibrium quantities traded by the price informed agent.

Corollary 9 The equilibrium price when there is no supply informed agent is

$$
\begin{aligned}
\widetilde{p}_{I}= & \frac{2 \sigma_{e}^{2}}{N \sigma_{v}^{2}+2 \sigma_{e}^{2}} \bar{v}+\frac{\sigma_{v}^{2}}{N \sigma_{v}^{2}+2 \sigma_{e}^{2}} \sum_{n=1}^{N} \widetilde{i}_{n}-\frac{\sigma_{v}^{2}}{N \sigma_{v}^{2}+2 \sigma_{e}^{2}}\left(\frac{N(N-1) \sigma_{e}^{2}}{(N-2) \sigma_{S}^{2}}\right) \widetilde{S} \\
& -\frac{\sigma_{v}^{2}}{\left(N \sigma_{v}^{2}+2 \sigma_{e}^{2}\right)(N-1)}\left(\frac{N(N-1) \sigma_{e}^{2}}{(N-2) \sigma_{S}^{2}}\right)^{1 / 2} \bar{m} .
\end{aligned}
$$

Notice that the price is here also an unbiased estimator of $\widetilde{v}$ if and only if $\bar{m}=0$. Next we compute the same market indicators we have computed for the economy with a supply informed agent. An interesting remark to be made is that neither the volatility of prices nor the efficiency of prices depend on the shocks in supply. 
Corollary 10 The market indicators for an economy without a supply informed agent are the following:

1) The price volatility, measured as the variance of price, is

$$
\operatorname{Var}\left(\widetilde{p}_{I}\right)=N\left(\frac{\sigma_{v}^{2}}{N \sigma_{v}^{2}+2 \sigma_{e}^{2}}\right)^{2}\left(\sigma_{v}^{2}+\frac{(2 N-3)}{(N-2)} \sigma_{e}^{2}\right) .
$$

2) The information content of prices is

$$
\operatorname{Var}(\widetilde{v})-\operatorname{Var}\left(\widetilde{v} \mid \widetilde{p_{I}}\right)=N \sigma_{v}^{2}(N-2)\left((N-2) \sigma_{v}^{2}+(2 N-3) \sigma_{e}^{2}\right)^{-1} .
$$

3) The expected volume traded by a price informed agent is

$$
E\left(\left|x_{I, n}\right|\right)=\frac{1}{N} \bar{m}+\left(\frac{2}{\pi}\right)^{1 / 2}\left(\sigma_{S}^{2} \frac{\left((N-2) \sigma_{v}^{2}+(N-1) \sigma_{e}^{2}\right)}{N^{2} \sigma_{e}^{2}}\right) .
$$

4) The expected profit of a price informed agent is

$$
\Pi_{I, n}^{P I}=E\left(\widetilde{\pi}_{I, n}^{P I}\right)=E\left(\left(\widetilde{v}-\widetilde{p}_{I}\right) \widetilde{x}_{n}\right)=\frac{\sigma_{v}^{2}}{N\left(N \sigma_{v}^{2}+2 \sigma_{e}^{2}\right)}\left(\frac{N(N-1) \sigma_{e}^{2}}{(N-2) \sigma_{S}^{2}}\right)^{1 / 2}\left(\frac{\bar{m}^{2}}{N-1}+\sigma_{S}^{2}\right) .
$$

\section{Comparison of M arket Indicators}

We are comparing now the market indicators in the case there exists a supply informed agent with the case there is no supply informed agent. We study first the effect the presence of the supply informed agent brings about on the market depth. We have that

$$
\gamma \equiv N \gamma^{P I}+\gamma^{S I}=\frac{\left(N^{2} \sigma_{v}^{2}+(2 N-1) \sigma_{e}^{2}\right) \sigma_{e}}{2 N^{2} \sigma_{v}^{2}\left(N \sigma_{v}^{2}+\sigma_{e}^{2}\right) \sigma_{S}}\left(\frac{\left(N(N-2) \sigma_{v}^{2}-\sigma_{e}^{2}\right)\left(N^{2} \sigma_{v}^{2}+\sigma_{e}^{2}\right)}{(N-1)}\right)^{1 / 2}
$$

the market depth in the case we have a supply informed agent and

$$
\gamma_{P I} \equiv N \gamma_{I}=\frac{\left(N \sigma_{v}^{2}+2 \sigma_{e}^{2}\right) \sigma_{S}}{\sigma_{v}^{2} \sigma_{e}}\left(\frac{(N-2)}{N(N-1)}\right)
$$

the market depth in the case we do not have any supply informed agent. The market depth is smaller in the case we have a supply informed agent in the market $\gamma<\gamma_{P I}$. This result is quite intuitive if we think that the supply informed agent plays a dual 
role in the market. First, he reveals himself a part of his information in the process of trading. Second, by having the information about supply he determines the price informed agents to reveal more of the information they own.

An interesting result that we obtain is that when there exists a supply informed trader in the market the price informed traders are trading more aggressively on their private information $\left(\beta^{P I}>\beta_{I}\right)$ but they devote less to the market making activity

$$
\omega^{P I}=\frac{\sigma_{e}^{2}\left(N(3 N-2) \sigma_{v}^{2}+(2 N-1) \sigma_{e}^{2}\right) \delta^{1 / 2}}{2 N^{2} \sigma_{v}^{2}\left(N^{2} \sigma_{v}^{2}+\sigma_{e}^{2}\right)\left(N \sigma_{v}^{2}+\sigma_{e}^{2}\right)}<\omega_{I}=\frac{2 \sigma_{e}^{2}}{N \sigma_{v}^{2}}\left(\frac{(N-2) \sigma_{S}^{2}}{N(N-1) \sigma_{e}^{2}}\right) .
$$

The inside information allows the price informed agents to make gains on the expense of the market makers. However, when there exists a supply informed agent who has the ability to disentangle the order flow originated by price informed agents from a shock in supply, the advantage of the price informed agent diminishes and therefore, his market making gains. A part of the gains that the price informed agents where making from market maker activity are now made by the supply informed agent. As we have seen already the price informed agents still put a higher weight on the maker making activity than the supply informed agent does. This tells us that a dealer although he might have information about supply faces strong competition in market making from the other traders. Moreover, we have that the effect of trading more aggressively on their information dominates the effect of decreasing the market maker activity and this leads to a higher trading volume by price informed agents.

Proposition 3 The presence of the supply informed agent in the market leads to higher volatility of prices, higher informativeness of prices and higher volume of trading by price informed agents.

The results that the volatility and informativeness of prices increase in the case there exists a supply informed agent is due to two factors. First, the existence in the market of the information about supply forces informed agents to reveal more of their information. But also, the shock in supply affects more the price than in the case there is no supply informed agent because the price informed agents get some information about supply from the action of the supply informed trader. 


\section{Conclusions}

In this paper we have presented a model of insider trading where the agents might have information either about prices or about supply. This information is aggregated and partially revealed through the equilibrium price, so the agents will end up with more information than they initially posses. Our goal is twofold. First we try to understand how the presence in the market of a supply informed agent and the interaction with the price informed agents can change the behaviour of the price informed agents and the structure of the market. Then, we see how the shocks in different components of supply can alter the market structure, the price formation and the behaviour of the agents, and therefore the impact of this shocks in the equilibrium outcome.

We consider an imperfectly competitive rational expectations setup and characterize the Bayesian Nash equilibrium in demand schedules. We characterize in closed form the symmetric linear equilibrium for the case the errors of the signals of the price informed agents are noncorrelated. Allowing the supply informed agent to behave strategically, he makes positive profits (unlike in the perfect competitive case) and increases the amount of information revealed in prices. We see that he has a dual role in inducing information transmission in the market: first because he owns superior information which he reveals in the trading process and second, because he urges the price informed agent to reveal more of their information. Hower, the most important consequence of his presence in the market is that he decreases market liquidity ( this outcome being brought about the strategic behavior and the mechanism of information transmission through prices).

We have also studied how the market performance is affected in our model by the quality of information received by the agents. The comparative statics results about market liquidity measured as market depth tell us that it is decreasing in the variance of the error of the signal received by price informed agents, increasing in the variance of the supply shock known only by the supply informed agent and has an inverted $U$ shape as respect to the variance of the liquidation value. Comparing the market indicators in our model with the ones in the benchmark case (where there is no supply informed agent) we conclude that the supply informed agent does indeed have 
an important effect. We find that the market depth decreases, while the volatility of prices, informativeness of prices and intensity of trading of price informed agents all increase.

We have considered also the case when the supply informed agent has information only about a component of supply. This setup is similar to the one in Gennotte and Leland (1990), where the supply has three components: a component known by everyone, a component known by the supply informed agent and another one known by nobody. The numerical analysis we have performed for this case suggests a similar pattern. However, in this case the supply informed agent will not put always a positive weight on price. Since he cannot anymore disentangle perfectly the two factors that might affect the prices (the news about the liquidation value of the asset revealed by the price informed agents or a shock in the unknown component of supply), he will not have anymore the same effect on market liquidity. However, for relative high variance

of the known component in supply relative to the unknown component, $\frac{\sigma_{S}}{\sigma_{L}}$ the result we have obtained here will still hold.

Finally, we would like to extend our work in modelling the process of information aquisition in a similar way to Froot et al. (1992). They develop a model à la Kyle (1985) were the informed traders have the possibility to acquire information about two different components of the liquidation value of the asset and show that the traders may herd on the same information trying to learn what other traders also know.

\section{A Appendix}

Lemma A.1 In a symmetric linear equilibrium $N \gamma^{P I}+\gamma^{S I} \neq 0$.

Proof. We look for a symmetric linear equilibrium. Therefore, we use the linear strategies defined in (2) and we can write the market clearing condition (1) as it follows:

$$
N \alpha^{P I}+\beta^{P I} \sum_{n=1}^{N} \widetilde{i_{n}}-N \gamma^{P I} \widetilde{p}+\alpha^{S I}+\beta^{S I} \widetilde{S}-\gamma^{S I} \widetilde{p}=\bar{m}+\widetilde{S}
$$


We define $\gamma \equiv N \gamma^{P I}+\gamma^{S I}$ and $\alpha \equiv N \alpha^{P I}+\alpha^{S I}$. Using these definitions, the market clearing condition can be written as

$$
\alpha+\beta^{P I} \sum_{n=1}^{N} \widetilde{i_{n}}-\gamma \widetilde{p}-\left(1-\beta^{S I}\right) \widetilde{S}=\bar{m}
$$

We want to prove that $\gamma \neq 0$. Let us suppose that $\gamma=0$. Then, the above condition becomes

$$
\alpha+\beta^{P I} \sum_{n=1}^{N} \widetilde{i_{n}}-\left(1-\beta^{S I}\right) \widetilde{S}=\bar{m} .
$$

Since $\widetilde{i_{n}}, n=1, \ldots, N$ are independent of $\widetilde{S}$, it results that $\beta^{P I}=0$. Plugging it in the above equation we obtain that

$$
\alpha-\left(1-\beta^{S I}\right) \widetilde{S}=\bar{m}
$$

which cannot be satisfied because $\alpha$ and $\bar{m}$ are real numbers and $\widetilde{S}$ is a random variable. We obtained therefore, a contradiction.

Lemma A.2 In a symmetric linear equilibrium the optimal demand for the price informed trader $n$ and for the supply informed trader are, respectively,

$$
\begin{aligned}
x_{n}\left(\widetilde{p}, \widetilde{i_{n}}\right) & =\left((N-1) \gamma^{P I}+\gamma^{S I}\right)\left[E\left(\widetilde{v} \mid \widetilde{p}, \widetilde{i_{n}}\right)-\widetilde{p}\right] \\
y(\widetilde{p}, \widetilde{S}) & =N \gamma^{P I}[E(\widetilde{v} \mid \widetilde{p}, \widetilde{S})-\widetilde{p}]
\end{aligned}
$$

with $\gamma^{P I}>0$, and $(N-1) \gamma^{P I}+\gamma^{S I}>0$.

P roof. Let us first determine the optimal demand for the price informed traders. Price informed trader $n$ considers the other players' strategies as given by (2). As a result, he is facing the following residual demand:

$$
p=\frac{\alpha-\alpha^{P I}+\beta^{P I} \sum_{j \neq n} \widetilde{i_{j}}-\left(1-\beta^{S I}\right) \widetilde{S}-\bar{m}}{(N-1) \gamma^{P I}+\gamma^{S I}}+\frac{x_{n}}{(N-1) \gamma^{P I}+\gamma^{S I}}
$$


and he solves the following maximization problem:

$$
\begin{aligned}
& \max _{x_{\mathrm{n}} \in \mathrm{R}} E\left((\widetilde{v}-\widetilde{p}) x_{n} \mid \widetilde{p}, \widetilde{i_{n}}\right) \Leftrightarrow \\
& \max _{x_{\mathrm{n}} \in \mathrm{R}} E\left(\left(\widetilde{v}-\frac{\alpha-\alpha^{P I}+\beta^{P I} \sum_{j \neq n} \widetilde{i_{j}}-\left(1-\beta^{S I}\right) \widetilde{S}-\bar{m}-x_{n}}{(N-1) \gamma^{P I}+\gamma^{S I}}\right) x_{n} \mid \widetilde{p}, \widetilde{i_{n}}\right) .
\end{aligned}
$$

The first order condition for this problem is

$$
\begin{aligned}
E\left(\widetilde{v} \mid \widetilde{p}, \widetilde{i_{n}}\right)- & E\left(\frac{\alpha-\alpha^{P I}+\beta^{P I} \sum_{j \neq n} \tilde{i_{j}}-\left(1-\beta^{S I}\right) \widetilde{S}-\bar{m}}{(N-1) \gamma^{P I}+\gamma^{S I}} \mid \widetilde{p}, \widetilde{i_{n}}\right) \\
& -\frac{2 x_{n}}{(N-1) \gamma^{P I}+\gamma^{S I}}=0 .
\end{aligned}
$$

Using (9) we can write further (10) as

$$
E\left(\widetilde{v} \mid \widetilde{p}, \widetilde{i_{n}}\right)-p-\frac{x_{n}}{(N-1) \gamma^{P I}+\gamma^{S I}}=0
$$

and from here we find the optimal demand of price informed trader $n$ :

$$
x_{n}=\left((N-1) \gamma^{P I}+\gamma^{S I}\right)\left(E\left(\widetilde{v} \mid \widetilde{p}, \widetilde{i_{n}}\right)-p\right) .
$$

The second order sufficient condition for this maximization problem is

$$
-\frac{2}{(N-1) \gamma^{P I}+\gamma^{S I}}<0 \Leftrightarrow(N-1) \gamma^{P I}+\gamma^{S I}>0 .
$$

Similarly, the supply informed trader takes as given the strategies of the price informed traders and in conformity with (2). The residual demand faced by him is therefore

$$
p=\frac{N \alpha^{P I}+N \beta^{P I} \widetilde{v}+\beta^{P I} \sum_{n=1}^{N} \widetilde{e_{n}}-\bar{m}-\widetilde{S}}{N \gamma^{P I}}+\frac{y}{N \gamma^{P I}} .
$$

The supply informed trader solves the following maximization problem:

$$
\begin{aligned}
& \max _{y \in \mathrm{R}} E((\widetilde{v}-\widetilde{p}) y \mid \widetilde{p}, \widetilde{S}) \Leftrightarrow \\
& \max _{y \in \mathrm{R}} E\left(\left(\widetilde{v}-\frac{N \alpha^{P I}+N \beta^{P I} \widetilde{v}+\beta^{P I} \sum_{n=1}^{N} \widetilde{e_{n}}-\bar{m}-\widetilde{S}}{N \gamma^{P I}}+\frac{y}{N \gamma^{P I}}\right) y \mid \widetilde{p}, \widetilde{S}\right) .
\end{aligned}
$$


The first order condition for this problem is

$$
E(\widetilde{v} \mid \widetilde{p}, \widetilde{S})-E\left(\frac{N \alpha^{P I}+N \beta^{P I} \widetilde{v}+\beta^{P I} \sum_{n=1}^{N} \widetilde{e_{j}}-\bar{m}-\widetilde{S}-\widetilde{L}}{N \gamma^{P I}} \mid \widetilde{p}, \widetilde{S}\right)-\frac{2 y}{N \gamma^{P I}}=0 .
$$

Using (11) we can write further (12) as

$$
E(\widetilde{v} \mid \widetilde{p}, \widetilde{S})-p-\frac{y}{N \gamma^{P I}}=0
$$

and from here we find the optimal demand of supply informed trader

$$
y=N \gamma^{P I}(E(\widetilde{v} \mid \widetilde{p}, \widetilde{S})-p) .
$$

The second order sufficient condition for this maximization problem is

$$
-\frac{2}{N \gamma^{P I}}<0 \Leftrightarrow N \gamma^{P I}>0 .
$$

Since $N \geq 1$ it results $\gamma^{P I}>0$.

Lemma A.3 In a symmetric linear equilibrium for any $n=1, \ldots, N$ we have

$$
\begin{aligned}
E\left(\widetilde{v} \mid \widetilde{p}=p, \widetilde{i_{n}}=i_{n}\right)= & \bar{v}\left(1-A(N-1) \beta^{P I}-B\right)-A(\alpha-\bar{m}) \\
& +\left(B-A \beta^{P I}\right) \tilde{i_{n}}+A \gamma \widetilde{p} .
\end{aligned}
$$

Proof. We can rewrite the market clearing condition (6) as

$$
\widetilde{p} \gamma-\alpha+\bar{m}-\beta^{P I} \widetilde{i_{n}}=(N-1) \beta^{P I} \widetilde{v}+\beta^{P I} \sum_{j \neq n} \widetilde{e_{j}}-\left(1-\beta^{S I}\right) \widetilde{S}
$$

From here it results that $\left(\widetilde{p}, \widetilde{i_{n}}\right)$ is informationally equivalent to $\left(\widetilde{h_{n}}, \widetilde{i_{n}}\right)$ where by definition $\widetilde{h_{n}} \equiv(N-1) \beta^{P I} \widetilde{v}+\beta^{P I} \sum_{j \neq n} \widetilde{e_{j}}-\left(1-\beta^{S I}\right) \widetilde{S}$. Consequently, we have $E\left(\widetilde{v} \mid \widetilde{p}=p, \widetilde{i_{n}}=i_{n}\right)=E\left(\widetilde{v} \mid \widetilde{h_{n}}=h_{n}, \widetilde{i_{n}}=i_{n}\right)$. Applying the projection theorem for normally distributed random variables we obtain that

$$
E\left(\widetilde{v} \mid \widetilde{h_{n}}=h_{n}, \widetilde{i_{n}}=i_{n}\right)=\bar{v}+\left(\begin{array}{cc}
A & B
\end{array}\right)\left(\begin{array}{c}
\widetilde{h_{n}}-E\left(\widetilde{h_{n}}\right) \\
\widetilde{i_{n}}-E\left(\widetilde{i_{n}}\right)
\end{array}\right)
$$


where $\left(\begin{array}{cc}A & B\end{array}\right)=\operatorname{cov}\left(\widetilde{v},\left(\widetilde{h_{n}}, \widetilde{i_{n}}\right)\right)\left(\operatorname{var}\left(\widetilde{h_{n}}, \widetilde{i_{n}}\right)\right)^{-1}$, when $\left(\operatorname{var}\left(\widetilde{h_{n}}, \widetilde{i_{n}}\right)\right)^{-1}$ exists. We compute $\operatorname{cov}\left(\widetilde{v}, \widetilde{h_{n}}\right)=\operatorname{cov}\left(\widetilde{v},(N-1) \beta^{P I} \widetilde{v}+\beta^{P I} \sum_{j \neq n} \widetilde{\xi}_{j}-\left(1-\beta^{S I}\right) \widetilde{S}\right)=$ $(N-1) \beta^{P I} \sigma_{v}^{2}$. Hence, we have that $\operatorname{cov}\left(\widetilde{v},\left(\widetilde{h_{n}}, \widetilde{i_{n}}\right)\right)=\left(\operatorname{cov}\left(\widetilde{v}, \widetilde{h_{n}}\right), \operatorname{cov}\left(\left(\widetilde{v}, \widetilde{i_{n}}\right)\right)\right)=$ $\left((N-1) \beta^{P I} \sigma_{v}^{2}, \sigma_{v}^{2}\right)$. Then we calculate the variance matrix. We calculate firstly

$$
\begin{aligned}
\operatorname{var}\left(\widetilde{h_{n}}\right) & =\operatorname{var}\left((N-1) \beta^{P I} \widetilde{v}+\beta^{P I} \sum_{j \neq n} \widetilde{e_{j}}-\left(1-\beta^{S I}\right) \widetilde{S}\right)= \\
& =\left(\beta^{P I}\right)^{2}(N-1)\left((N-1) \sigma_{v}^{2}+\sigma_{e}^{2}\right)+\left(1-\beta^{S I}\right)^{2} \sigma_{S}^{2} .
\end{aligned}
$$

In order to simplify the notation we define $q \equiv(N-1)\left((N-1) \sigma_{v}^{2}+\sigma_{e}^{2}\right)$. Next we see that $\operatorname{cov}\left(\widetilde{h_{n}}, \widetilde{i_{n}}\right)=(N-1) \beta^{P I} \sigma_{v}^{2}$ and consequently, we can write the variance matrix as

$$
\operatorname{var}\left(\left(\widetilde{h_{n}}, \widetilde{i_{n}}\right)\right)=\left(\begin{array}{cc}
\left(\beta^{P I}\right)^{2} q+\left(1-\beta^{S I}\right)^{2} \sigma_{S}^{2} & (N-1) \beta^{P I} \sigma_{v}^{2} \\
(N-1) \beta^{P I} \sigma_{v}^{2} & \sigma_{v}^{2}+\sigma_{e}^{2}
\end{array}\right)
$$

The determinant of the variance matrix is

$$
M=\left(\beta^{P I}\right)^{2}(N-1)\left(N \sigma_{v}^{2}+\sigma_{e}^{2}\right) \sigma_{e}^{2}+\left(1-\beta^{S I}\right)^{2} \sigma_{S}^{2}\left(\sigma_{v}^{2}+\sigma_{e}^{2}\right)
$$

and this is always higher than zero.

Since $M \neq 0$, it exists the inverse of the variance matrix and it equals to

$$
\left(\operatorname{var}\left(\widetilde{h_{n}}, \widetilde{i_{n}}\right)\right)^{-1}=\frac{1}{M}\left(\begin{array}{cc}
\sigma_{v}^{2}+\sigma_{e}^{2} & -(N-1) \beta^{P I} \sigma_{v}^{2} \\
-(N-1) \beta^{P I} \sigma_{e}^{2} & \left(\beta^{P I}\right)^{2} q+\left(1-\beta^{S I}\right)^{2} \sigma_{S}^{2}
\end{array}\right)
$$

Once we have calculated $\operatorname{cov}\left(\widetilde{v},\left(\widetilde{h_{n}}, \widetilde{i_{n}}\right)\right)$ and $\left(\operatorname{var}\left(\widetilde{h_{n}}, \widetilde{i_{n}}\right)\right)^{-1}$ we can proceed and identify $A$ and $B$. It results that

$$
\begin{aligned}
& A=M^{-1}(N-1) \beta^{P I} \sigma_{v}^{2} \sigma_{e}^{2} \text { and } \\
& B=M^{-1}\left[\left(\beta^{P I}\right)^{2}(N-1) \sigma_{v}^{2} \sigma_{e}^{2}+\left(1-\beta^{S I}\right)^{2} \sigma_{S}^{2} \sigma_{v}^{2}\right] .
\end{aligned}
$$

Since $\widetilde{h_{n}} \equiv(N-1) \beta^{P I} \widetilde{v}+\beta^{P I} \sum_{j \neq n} \widetilde{e_{j}}-\left(1-\beta^{S I}\right) \widetilde{S}$ we have $E\left(\widetilde{h_{n}}\right)=(N-1) \beta^{P I} \bar{v}$. In addition, we assumed that $E\left(\widetilde{i_{n}}\right)=E\left(\widetilde{v}+\widetilde{e_{n}}\right)=\bar{v}$. Using the above values for 
expectations and the formula (13) for $\widetilde{h_{n}}$ the expression (14) can be written as

$$
\begin{aligned}
E & \left(\widetilde{v} \mid \widetilde{h_{n}}=h_{n}, \widetilde{i_{n}}=i_{n}\right)=\bar{v}+A\left(\widetilde{h_{n}}-(N-1) \beta^{P I} \bar{v}\right)+B\left(\widetilde{i_{n}}-\bar{v}\right) \\
& =\bar{v}\left(1-A(N-1) \beta^{P I}-B\right)-A(\alpha-\bar{m})+\left(B-A \beta^{P I}\right) \widetilde{i_{n}}+A \gamma \widetilde{p}
\end{aligned}
$$

where $A$ and $B$ are given by (15).

Lemma A.4 In a symmetric linear equilibrium we have

$$
E(\widetilde{v} \mid \widetilde{p}=p, \widetilde{S}=S)=\bar{v}\left(1-C N \beta^{P I}\right)-C(\alpha-\bar{m})+\left(1-\beta^{S I}\right) C \widetilde{S}+C \gamma \widetilde{p} .
$$

Proof. We write again the market clearing condition (6) this time in order to find a pair informationally equivalent to $(\widetilde{p}, \widetilde{S})$

$$
\widetilde{p} \gamma-\alpha+\bar{m}+\left(1-\beta^{S I}\right) \widetilde{S}=\beta^{P I} \sum_{n=1}^{N} \widetilde{i_{n}}
$$

We define $\theta \equiv \beta^{P I} \sum_{n=1}^{N} \widetilde{i_{n}}$. From here it results that $(\widetilde{\theta}, \widetilde{S})$ is informationally equivalent to $(\widetilde{p}, \widetilde{S})$. Consequently, $E(\widetilde{v} \mid \widetilde{p}=p, \widetilde{S}=S)=E(\widetilde{v} \mid \widetilde{\theta}=\theta, \widetilde{S}=S)$. Applying again the projection theorem for normally distributed random variables we obtain that

$$
E(\widetilde{v} \mid \widetilde{\theta}=\theta, \widetilde{S}=S)=\bar{v}+(C, D)\left(\begin{array}{c}
\widetilde{\theta}-E(\widetilde{\theta}) \\
\widetilde{S}-E(\widetilde{S})
\end{array}\right)
$$

where $(C, \quad D)=\operatorname{cov}(\widetilde{v},(\widetilde{\theta}, \widetilde{S}))(\operatorname{var}(\widetilde{\theta}, \widetilde{S}))^{-1}$.

Let us calculate $\operatorname{cov}(\widetilde{v},(\widetilde{\theta}, \widetilde{S}))$. First we compute the covariance of $\widetilde{v}$ and $\widetilde{\theta}$ $\operatorname{cov}(\widetilde{v}, \widetilde{\theta})=\operatorname{cov}\left(\widetilde{v}, N \beta^{P I} \widetilde{v}+\beta^{P I} \sum_{n=1}^{N} \widetilde{e_{n}}\right)=N \beta^{P I} \sigma_{v}^{2}$. Since $\widetilde{v}$ and $\widetilde{S}$ are independent random variables, it results that $\operatorname{cov}(\widetilde{v},(\widetilde{\theta}, \widetilde{S}))=\left(N \beta^{P I} \sigma_{v}^{2}, \quad 0\right)$. Similarly we calculate the variance-covariance matrix. First, we calculate $\operatorname{cov}(\widetilde{\theta}, \widetilde{\theta})=$ $\operatorname{cov}\left(N \beta^{P I} \widetilde{v}+\beta^{P I} \sum_{n=1}^{N} \widetilde{e_{n}}, N \beta^{P I} \widetilde{v}+\beta^{P I} \sum_{n=1}^{N} \widetilde{e_{n}}\right)=\left(\beta^{P I}\right)^{2} N\left(N \sigma_{v}^{2}+\sigma_{e}^{2}\right)$. Then notice that $\operatorname{cov}(\widetilde{\theta}, \widetilde{S})=\operatorname{cov}\left(N \beta^{P I} \widetilde{v}+\beta^{P I} \sum_{n=1}^{N} \widetilde{e_{n}}, \widetilde{S}\right)=0$. It results that

$$
\operatorname{var}(\widetilde{\theta}, \widetilde{S})=\left(\begin{array}{cc}
\left(\beta^{P I}\right)^{2} N\left(N \sigma_{v}^{2}+\sigma_{e}^{2}\right) & 0 \\
0 & \sigma_{S}^{2}
\end{array}\right)
$$


The variance matrix is nonsingular and its inverse is

$$
(\operatorname{var}(\widetilde{\theta}, \widetilde{S}))^{-1}=\left(\begin{array}{cc}
\left(\left(\beta^{P I}\right)^{2} N\left(N \sigma_{v}^{2}+\sigma_{e}^{2}\right)\right)^{-1} & 0 \\
0 & \left(\sigma_{S}^{2}\right)^{-1}
\end{array}\right),
$$

and consequently,

$$
C=\sigma_{v}^{2}\left(\beta^{P I}\left(N \sigma_{v}^{2}+\sigma_{e}^{2}\right)\right)^{-1} \text { and } D=0 .
$$

Since $E\left(\widetilde{i_{n}}\right)=\bar{v}$, and $\widetilde{\theta} \equiv N \beta^{P I} \widetilde{v}+\beta^{P I} \sum_{n=1}^{N} \widetilde{e_{n}}$ we have that $E(\widetilde{\theta})=N \beta^{P I} \bar{v}$. In addition, we assumed that $E(\widetilde{S})=0$. Using the above values for expectations, the fact that $D=0$ and the formula (17) for $\widetilde{\theta}$, the expression (14) can be written as

$$
\begin{aligned}
& E(\widetilde{v} \mid \widetilde{\theta}=\theta, \widetilde{S}=S)=\bar{v}+C\left(\widetilde{\theta}-N \beta^{P I} \bar{v}\right)+D \widetilde{S} \\
& \quad=\bar{v}\left(1-C N \beta^{P I}\right)-C(\alpha-\bar{m})+\left(1-\beta^{S I}\right) C \widetilde{S}+C \gamma \widetilde{p},
\end{aligned}
$$

where $C$ is given by formula (19).

Lemma A.5 The coefficients $\alpha^{P I}, \beta^{P I}, \gamma^{P I}, \alpha^{S I}, \beta^{S I}, \gamma^{S I}$ are the solution of the following system of equations:

$$
\left\{\begin{aligned}
\alpha^{P I} & =\left((N-1) \gamma^{P I}+\gamma^{S I}\right)\left(\bar{v}\left(1-A(N-1) \beta^{P I}-B\right)-A(\alpha-\bar{m})\right) \\
\beta^{P I} & =\left((N-1) \gamma^{P I}+\gamma^{S I}\right)\left(B-A \beta^{P I}\right) \\
\gamma^{P I} & =\left((N-1) \gamma^{P I}+\gamma^{S I}\right)(1-A \gamma) \\
\alpha^{S I} & =N \gamma^{P I}\left(\bar{v}\left(1-C N \beta^{P I}\right)-C(\alpha-\bar{m})\right) \\
\beta^{S I} & =N \gamma^{P I}\left(\left(1-\beta^{S I}\right) C\right. \\
\gamma^{S I} & =N \gamma^{P I}(1-C \gamma) \\
M & =\left(\beta^{P I}\right)^{2}(N-1)\left(N \sigma_{v}^{2}+\sigma_{e}^{2}\right) \sigma_{e}^{2}+\left(1-\beta^{S I}\right)^{2} \sigma_{S}^{2}\left(\sigma_{v}^{2}+\sigma_{e}^{2}\right) \\
A & =M^{-1}(N-1) \beta^{P I} \sigma_{v}^{2} \sigma_{e}^{2} \\
B & =M^{-1}\left(\left(\beta^{P I}\right)^{2}(N-1) \sigma_{v}^{2} \sigma_{e}^{2}+\left(1-\beta^{S I}\right)^{2} \sigma_{S}^{2} \sigma_{v}^{2}\right) \\
C & =\sigma_{v}^{2}\left(\beta^{P I}\left(N \sigma_{v}^{2}+\sigma_{e}^{2}\right)\right)^{-1} .
\end{aligned}\right.
$$

Proof of Lemma A.5. In Lemma A.3 and Lemma A.4 for we have established the expressions for $E\left(\widetilde{v} \mid \widetilde{p}=p, \widetilde{i_{n}}=i_{n}\right)$ and $E(\widetilde{v} \mid \widetilde{p}=p, \widetilde{S}=S)$. We will use them 
now to find the expressions for the strategies for the price informed agents and for the supply informed agent.

First, since $E\left(\widetilde{v} \mid \widetilde{p}=p, \widetilde{i_{n}}=i_{n}\right)=E\left(\widetilde{v} \mid \widetilde{h_{n}}=h_{n}, \widetilde{i_{n}}=i_{n}\right)$ we plug (16) in (7) and we obtain that

$$
\begin{aligned}
x_{n}\left(\widetilde{p}, \tilde{i_{n}}\right)= & \left((N-1) \gamma^{P I}+\gamma^{S I}\right)\left(\bar{v}\left(1-A(N-1) \beta^{P I}-B\right)-A(\alpha-\bar{m})\right. \\
& \left.+\left(B-A \beta^{P I}\right) \widetilde{i_{n}}+(A \gamma-1) \widetilde{p}\right)
\end{aligned}
$$

We identify the coefficients in the definition of the strategy of the price informed trader $n$ (2) and we get the following equations:

$$
\begin{aligned}
& \alpha^{P I}=\left((N-1) \gamma^{P I}+\gamma^{S I}\right)\left(\bar{v}\left(1-A(N-1) \beta^{P I}-B\right)-A(\alpha-\bar{m})\right) \\
& \beta^{P I}=\left((N-1) \gamma^{P I}+\gamma^{S I}\right)\left(B-A \beta^{P I}\right) \\
& \gamma^{P I}=\left((N-1) \gamma^{P I}+\gamma^{S I}\right)(1-A \gamma),
\end{aligned}
$$

where $A$ and $B$ are given by (15).

Second, since $E(\widetilde{v} \mid \widetilde{p}=p, \widetilde{S}=S)=E(\widetilde{v} \mid \widetilde{\theta}=\theta, \widetilde{S}=S)$ we plug (20) in (8) and we obtain in a similar manner

$$
y(\widetilde{p}, \widetilde{S})=N \gamma^{P I}\left(\bar{v}-C(\alpha-\bar{m})+\left(1-\beta^{S I}\right) C \widetilde{S}+(C \gamma-1) \widetilde{p}\right)
$$

We identify the coefficients in the definition of the strategy of the supply informed trader (2) and we get the following equations:

$$
\begin{aligned}
& \alpha^{S I}=N \gamma^{P I}\left(\bar{v}\left(1-C N \beta^{P I}\right)-C(\alpha-\bar{m})\right) \\
& \beta^{S I}=N \gamma^{P I}\left(1-\beta^{S I}\right) C \\
& \gamma^{S I}=N \gamma^{P I}(1-C \gamma),
\end{aligned}
$$

where $C$ is given by (19).

Putting together the equations (15), (21), (19) and (22) we obtain that $\alpha^{P I}, \beta^{P I}$, $\gamma^{P I}, \alpha^{S I}, \beta^{S I}, \gamma^{S I}$ are the solution of the above system of equations.

Proof of Proposition 1. We leave apart the equations for $\alpha^{P I}$ and $\alpha^{S I}$ since these variables are not involved in the other equations. Then since by definition $\gamma=$ 
$N \gamma^{P I}+\gamma^{S I}$ we can write the equation

$$
\beta^{P I}=\left((N-1) \gamma^{P I}+\gamma^{S I}\right)\left(B-A \beta^{P I}\right)
$$

as

$$
\beta^{P I}=\left(\gamma-\gamma^{P I}\right)\left(B-A \beta^{P I}\right)
$$

and from here to solve for $\beta^{P I}$

$$
\beta^{P I}=\frac{\left(\gamma-\gamma^{P I}\right) B}{1+A\left(\gamma-\gamma^{P I}\right)}
$$

Similarly, we have that

$$
\gamma^{P I}=\left((N-1) \gamma^{P I}+\gamma^{S I}\right)(1-A \gamma)=\left(\gamma-\gamma^{P I}\right)(1-A \gamma)
$$

and we obtain from here that

$$
\gamma^{P I}=\frac{\gamma(1-A \gamma)}{2-A \gamma} .
$$

By substituting $\gamma^{P I}$ given by (24) in (23) we can write further $\beta^{P I}$ only as a function of $\gamma$ and $A$,

$$
\beta^{P I}=\frac{\left(\gamma-\frac{\gamma(1-A \gamma)}{2-A \gamma}\right) B}{1+A\left(\gamma-\frac{\gamma(1-A \gamma)}{2-A \gamma}\right)}=\frac{B \gamma}{2} .
$$

We obtain the coefficients for the supply informed agent in a similar way. We have that

$$
\beta^{S I}=N \gamma^{P I}\left(1-\beta^{S I}\right) C
$$

and from here it results that

$$
\beta^{S I}=\frac{N \gamma^{P I} C}{1+N \gamma^{P I} C} .
$$

Finally, using the formula (24) we obtain that

$$
\gamma^{S I}=N \gamma^{P I}(1-C \gamma)=\frac{N \gamma(1-A \gamma)(1-C \gamma)}{(2-A \gamma)} .
$$


By definition $\gamma=N \gamma^{P I}+\gamma^{S I}$. Then by replacing the formulas for $\gamma^{P I}$ and $\gamma^{S I}$ obtained before we obtain the following equation in $A, C$ and $\gamma$ :

$$
\frac{2-A \gamma}{1-A \gamma}=N(2-C \gamma)
$$

In this equation we replace $\gamma=\frac{2 \beta^{P I}}{B}$ and we obtain further

$$
\frac{B-A \beta^{P I}}{B-2 A \beta^{P I}}=N\left(1-\frac{1}{B} \frac{\sigma_{v}^{2}}{N \sigma_{v}^{2}+\sigma_{e}^{2}}\right) \text {. }
$$

Using the same equation we can rewrite $\beta^{S I}$ given by (26) in a simpler way.

$$
\beta^{S I}=\frac{N \gamma^{P I} C}{1+N \gamma^{P I} C}=\frac{N C \frac{\gamma(1-A \gamma)}{2-A \gamma}}{1+N C \frac{\gamma(1-A \gamma)}{2-A \gamma}}=\frac{C \frac{\gamma}{2-C \gamma}}{1+C \frac{\gamma}{2-C \gamma}}=\frac{C \gamma}{2}
$$

Next, we define $z \equiv\left(1-\beta^{S I}\right)^{2} \sigma_{S}^{2}$. Using (25), (29) and the equation in the system that defines $C$ we can write

$$
z=\left(\frac{2-C \gamma}{2}\right)^{2} \sigma_{S}^{2}=\left(1-\frac{C \beta^{P I}}{B}\right)^{2} \sigma_{S}^{2}=\left(1-\frac{1}{B} \frac{\sigma_{v}^{2}}{N \sigma_{v}^{2}+\sigma_{e}^{2}}\right)^{2} \sigma_{S}^{2} .
$$

Further on we compute the expressions for $A, B$ as function only of $\beta^{P I}$ and $z$. Thus

$$
A=\frac{(N-1) \beta^{P I} \sigma_{v}^{2} \sigma_{e}^{2}}{\left(\beta^{P I}\right)^{2}(N-1)\left(N \sigma_{v}^{2}+\sigma_{e}^{2}\right) \sigma_{e}^{2}+z\left(\sigma_{v}^{2}+\sigma_{e}^{2}\right)}
$$

By replacing it in the equation (28) we obtain

$$
\begin{aligned}
& \frac{B\left(\left(\beta^{P I}\right)^{2}(N-1)\left(N \sigma_{v}^{2}+\sigma_{e}^{2}\right) \sigma_{e}^{2}+z\left(\sigma_{v}^{2}+\sigma_{e}^{2}\right)\right)-(N-1)\left(\beta^{P I}\right)^{2} \sigma_{v}^{2} \sigma_{e}^{2}}{B\left(\left(\beta^{P I}\right)^{2}(N-1)\left(N \sigma_{v}^{2}+\sigma_{e}^{2}\right) \sigma_{e}^{2}+z\left(\sigma_{v}^{2}+\sigma_{e}^{2}\right)\right)-2(N-1)\left(\beta^{P I}\right)^{2} \sigma_{v}^{2} \sigma_{e}^{2}} \\
& =N\left(1-\frac{1}{B} \frac{\sigma_{v}^{2}}{N \sigma_{v}^{2}+\sigma_{e}^{2}}\right) .
\end{aligned}
$$

Let us define now as $u \equiv\left(\beta^{P I}\right)^{2}$ and $x=\left(1-\frac{1}{B} \frac{\sigma_{v}^{2}}{N \sigma_{v}^{2}+\sigma_{e}^{2}}\right)$. Then it results that $z=x^{2} \sigma_{S}^{2}$ and the above equation can be written as

$$
\frac{B\left(u(N-1)\left(N \sigma_{v}^{2}+\sigma_{e}^{2}\right) \sigma_{e}^{2}+x^{2} \sigma_{S}^{2}\left(\sigma_{v}^{2}+\sigma_{e}^{2}\right)\right)-(N-1) u \sigma_{v}^{2} \sigma_{e}^{2}}{B\left(u(N-1)\left(N \sigma_{v}^{2}+\sigma_{e}^{2}\right) \sigma_{e}^{2}+x^{2} \sigma_{S}^{2}\left(\sigma_{v}^{2}+\sigma_{e}^{2}\right)\right)-2(N-1) u \sigma_{v}^{2} \sigma_{e}^{2}}=N x .
$$


On the other hand, we have that

$$
\begin{aligned}
B= & \frac{\left(\beta^{P I}\right)^{2}(N-1) \sigma_{v}^{2} \sigma_{e}^{2}+z \sigma_{v}^{2}}{\left(\beta^{P I}\right)^{2}(N-1)\left(N \sigma_{v}^{2}+\sigma_{e}^{2}\right) \sigma_{e}^{2}+z\left(\sigma_{v}^{2}+\sigma_{e}^{2}\right)}= \\
& \frac{u(N-1) \sigma_{v}^{2} \sigma_{e}^{2}+x^{2} \sigma_{S}^{2} \sigma_{v}^{2}}{u(N-1)\left(N \sigma_{v}^{2}+\sigma_{e}^{2}\right) \sigma_{e}^{2}+x^{2} \sigma_{S}^{2}\left(\sigma_{v}^{2}+\sigma_{e}^{2}\right)} .
\end{aligned}
$$

We can now use this formula for $B$ to rewrite equation (30) as

$$
\frac{x^{2} \sigma_{S}^{2} \sigma_{v}^{2}}{x^{2} \sigma_{S}^{2} \sigma_{v}^{2}-(N-1) u \sigma_{v}^{2} \sigma_{e}^{2}}=N x .
$$

Since $\sigma_{v}^{2}>0$ and $\sigma_{S}^{2}>0$ this equation is equivalent to $x=0$ or $\frac{(N x-1) x \sigma_{S}^{2}}{N}=$ $(N-1) u \sigma_{e}^{2}$.

Replacing $(N-1) u \sigma_{e}^{2}=\frac{(N x-1) x \sigma_{S}^{2}}{N} \operatorname{in}(31)$ we get

$$
B=\frac{(N x-1) x \sigma_{S}^{2} \sigma_{v}^{2}+N x^{2} \sigma_{S}^{2} \sigma_{v}^{2}}{(N x-1) x \sigma_{S}^{2}\left(N \sigma_{v}^{2}+\sigma_{e}^{2}\right)+N x^{2} \sigma_{S}^{2}\left(\sigma_{v}^{2}+\sigma_{e}^{2}\right)} .
$$

But also, using the definition of $x$ we can write

$$
B=\frac{\sigma_{v}^{2}}{(1-x)\left(N \sigma_{v}^{2}+\sigma_{e}^{2}\right)} .
$$

Note that $1-x$ cannot be 0 since $\frac{1}{B} \frac{\sigma_{v}^{2}}{N \sigma_{v}^{2}+\sigma_{e}^{2}}>0$. So we have two expressions for $B$ and we equalize them obtaining the following equation in $x$ :

$$
\begin{aligned}
& \frac{\sigma_{v}^{2}}{(1-x)\left(N \sigma_{v}^{2}+\sigma_{e}^{2}\right)}=\frac{(N x-1) x \sigma_{S}^{2} \sigma_{v}^{2}+N x^{2} \sigma_{S}^{2} \sigma_{v}^{2}}{(N x-1) x \sigma_{S}^{2}\left(N \sigma_{v}^{2}+\sigma_{e}^{2}\right)+N x^{2} \sigma_{S}^{2}\left(\sigma_{v}^{2}+\sigma_{e}^{2}\right)}, \\
& \text { or equivalent } \frac{1}{(1-x)\left(N \sigma_{v}^{2}+\sigma_{e}^{2}\right)}=\frac{2 N x-1}{(N x-1)\left(N \sigma_{v}^{2}+\sigma_{e}^{2}\right)+N x\left(\sigma_{v}^{2}+\sigma_{e}^{2}\right)}
\end{aligned}
$$

This last equation has two solutions

$$
x=0 \text { and } x=\frac{(N+1)\left(N \sigma_{v}^{2}+\sigma_{e}^{2}\right)-N\left(\sigma_{v}^{2}+\sigma_{e}^{2}\right)}{2 N\left(N \sigma_{v}^{2}+\sigma_{e}^{2}\right)} .
$$

First, if $x=0$ then $z=x^{2} \sigma_{S}^{2}=0$ and it results $\beta^{S I}=1$.

Then $B=\frac{\sigma_{v}^{2}}{\left(N \sigma_{v}^{2}+\sigma_{e}^{2}\right)}$ and $u=0$. But $u=0$ implies $\beta^{P I}=0$ and from here $A=\gamma=0$ and the second order condition is not satisfied. 
Second, we have that

$$
x=\frac{(N+1)\left(N \sigma_{v}^{2}+\sigma_{e}^{2}\right)-N\left(\sigma_{v}^{2}+\sigma_{e}^{2}\right)}{2 N\left(N \sigma_{v}^{2}+\sigma_{e}^{2}\right)}=\frac{N^{2} \sigma_{v}^{2}+\sigma_{e}^{2}}{2 N\left(N \sigma_{v}^{2}+\sigma_{e}^{2}\right)}
$$

Using this formula we can compute then

$$
\begin{gathered}
\beta^{S I}=\frac{N^{2} \sigma_{v}^{2}+(2 N-1) \sigma_{e}^{2}}{2 N\left(N \sigma_{v}^{2}+\sigma_{e}^{2}\right)}, \\
B=\frac{2 N \sigma_{v}^{2}}{N^{2} \sigma_{v}^{2}+(2 N-1) \sigma_{e}^{2}} \text { and } \\
u=\frac{(N x-1) x \sigma_{S}^{2}}{N(N-1) \sigma_{e}^{2}}=\frac{\left(N(N-2) \sigma_{v}^{2}-\sigma_{e}^{2}\right)\left(N^{2} \sigma_{v}^{2}+\sigma_{e}^{2}\right) \sigma_{S}^{2}}{4\left(N \sigma_{v}^{2}+\sigma_{e}^{2}\right)^{2} N^{2}(N-1) \sigma_{e}^{2}}
\end{gathered}
$$

Notice that $\left(\beta^{P I}\right)^{2}=u$, so we need $u \geq 0$. If $N(N-2) \sigma_{v}^{2}-\sigma_{e}^{2}>0$ we have $u \geq 0$ and consequently, we have solution for $\beta^{P I}$ and it is equal to

$$
\beta^{P I}=\frac{1}{2 N\left(N \sigma_{v}^{2}+\sigma_{e}^{2}\right)} \sqrt{\frac{\left(N(N-2) \sigma_{v}^{2}-\sigma_{e}^{2}\right)\left(N^{2} \sigma_{v}^{2}+\sigma_{e}^{2}\right) \sigma_{S}^{2}}{(N-1) \sigma_{e}^{2}}} .
$$

Using the last equation in the system we can write also

$$
C=\frac{\sigma_{v}^{2}}{\beta^{P I}\left(N \sigma_{v}^{2}+\sigma_{e}^{2}\right)}=2 N \sigma_{v}^{2} \sqrt{\frac{(N-1) \sigma_{e}^{2}}{\left(N(N-2) \sigma_{v}^{2}-\sigma_{e}^{2}\right)\left(N^{2} \sigma_{v}^{2}+\sigma_{e}^{2}\right) \sigma_{S}^{2}}} .
$$

Next, since

$$
\beta^{S I}=N \gamma^{P I}\left(1-\beta^{S I}\right) C,
$$

we can find an expression for $\gamma^{P I}$. Using the formulas for $\beta^{S I}(32)$ and $C$ it results that

$$
\gamma^{P I}=\frac{\beta^{S I}}{N\left(1-\beta^{S I}\right) C}=\frac{N^{2} \sigma_{v}^{2}+(2 N-1) \sigma_{e}^{2}}{2 N^{2} \sigma_{v}^{2}} \sqrt{\frac{\left(N(N-2) \sigma_{v}^{2}-\sigma_{e}^{2}\right) \sigma_{S}^{2}}{(N-1) \sigma_{e}^{2}\left(N^{2} \sigma_{v}^{2}+\sigma_{e}^{2}\right)}} .
$$

Similarly,

$$
\gamma^{S I}=N \gamma^{P I}(1-C \gamma)=N \gamma^{P I}\left(1-2 \beta^{S I}\right)=-\frac{(N-1) \sigma_{e}^{2}}{\left(N \sigma_{v}^{2}+\sigma_{e}^{2}\right)} \gamma^{P I} .
$$


An important remark has to be made. The coefficient $\gamma^{S I}<0$, however the second order conditions are satisfied since

$$
(N-1) \gamma^{P I}+\gamma^{S I}=\frac{N(N-1) \sigma_{v}^{2}}{\left(N \sigma_{v}^{2}+\sigma_{e}^{2}\right)} \gamma^{P I}>0
$$

We compute now the ratio $\frac{\gamma^{P I}}{\beta^{P I}}$ because we will make use of it later on.

$$
\frac{\gamma^{P I}}{\beta^{P I}}=\frac{N^{2} \sigma_{v}^{2}+(2 N-1) \sigma_{e}^{2}}{N \sigma_{v}^{2}} \frac{\left(N \sigma_{v}^{2}+\sigma_{e}^{2}\right)}{\left(N^{2} \sigma_{v}^{2}+\sigma_{e}^{2}\right)}
$$

The only coefficients left to compute are $\alpha^{P I}$ and $\alpha^{S I}$. We have from the system that

$$
\begin{gathered}
\alpha^{P I}=\left((N-1) \gamma^{P I}+\gamma^{S I}\right)\left(\bar{v}\left(1-A(N-1) \beta^{P I}-B\right)-A(\alpha-\bar{m})\right) \\
\text { and } \alpha^{S I}=N \gamma^{P I}\left(\bar{v}\left(1-C N \beta^{P I}\right)-C(\alpha-\bar{m})\right)
\end{gathered}
$$

We will first compute $t \equiv(\alpha-\bar{m})$ and for that we use the definition $\alpha=N \alpha^{P I}+\alpha^{S I}$. Thus, we have

$$
(\alpha-\bar{m})=N\left(\frac{N(N-1) \sigma_{v}^{2}}{\left(N \sigma_{v}^{2}+\sigma_{e}^{2}\right)} \gamma^{P I}\right)(w-A(\alpha-\bar{m}))+N \gamma^{P I}(z-C(\alpha-\bar{m}))-\bar{m} .
$$

Solving for $t \equiv(\alpha-\bar{m})$ we obtain

$$
\begin{aligned}
t= & \frac{N \gamma^{P I} \bar{v}\left(N(N-1) \sigma_{v}^{2}\left(1-A(N-1) \beta^{P I}-B\right)+\left(1-C N \beta^{P I}\right)\left(N \sigma_{v}^{2}+\sigma_{e}^{2}\right)\right)}{\left(\left(N \sigma_{v}^{2}+\sigma_{e}^{2}\right)\left(1+N \gamma^{P I} C\right)+\left(N(N-1) \sigma_{v}^{2}\right) A N \gamma^{P I}\right)} \\
& -\frac{\bar{m}\left(N \sigma_{v}^{2}+\sigma_{e}^{2}\right)}{\left(\left(N \sigma_{v}^{2}+\sigma_{e}^{2}\right)\left(1+N \gamma^{P I} C\right)+\left(N(N-1) \sigma_{v}^{2}\right) A N \gamma^{P I}\right)}
\end{aligned}
$$

Using that

$$
\begin{aligned}
A \beta^{P I} & =\frac{\left(N(N-2) \sigma_{v}^{2}-\sigma_{e}^{2}\right)}{(N-1)\left(N^{2} \sigma_{v}^{2}+\sigma_{e}^{2}(2 N-1)\right)}, \\
B & =\frac{2 N \sigma_{v}^{2}}{N^{2} \sigma_{v}^{2}+(2 N-1) \sigma_{e}^{2}} \text { and } \\
C \beta^{P I} & =\frac{\sigma_{v}^{2}}{\left(N \sigma_{v}^{2}+\sigma_{e}^{2}\right)}
\end{aligned}
$$


we have that the denominator is

$$
\frac{N \gamma^{P I}}{\beta^{P I}} \sigma_{v}^{2}\left(\frac{2 N\left(N \sigma_{v}^{2}+\sigma_{e}^{2}\right)}{N^{2} \sigma_{v}^{2}+(2 N-1) \sigma_{e}^{2}}+N \frac{\left(N(N-2) \sigma_{v}^{2}-\sigma_{e}^{2}\right)}{\left(N^{2} \sigma_{v}^{2}+\sigma_{e}^{2}(2 N-1)\right)}\right)=\left(N \sigma_{v}^{2}+\sigma_{e}^{2}\right) N .
$$

and the numerator is

$$
\frac{N \gamma^{P I} \bar{v}\left((2 N-1) \sigma_{e}^{2}\left(N^{2} \sigma_{v}^{2}+\sigma_{e}^{2}\right)\right)}{N^{2} \sigma_{v}^{2}+\sigma_{e}^{2}(2 N-1)}-\bar{m}\left(N \sigma_{v}^{2}+\sigma_{e}^{2}\right)
$$

As a result,

$$
t=\frac{\gamma^{P I}\left((2 N-1) \sigma_{e}^{2}\left(N^{2} \sigma_{v}^{2}+\sigma_{e}^{2}\right)\right)}{\left(N^{2} \sigma_{v}^{2}+\sigma_{e}^{2}(2 N-1)\right)\left(N \sigma_{v}^{2}+\sigma_{e}^{2}\right)} \bar{v}-\frac{\bar{m}}{N} .
$$

We proceed now with the computations of the coefficients $\alpha^{P I}$ and $\alpha^{S I}$. As we have already seen

$$
\alpha^{P I}=\left((N-1) \gamma^{P I}+\gamma^{S I}\right)\left(\bar{v}\left(1-A(N-1) \beta^{P I}-B\right)-A t\right),
$$

and replacing the formulas we have obtained for $A, B$ and $t$ we obtain that

$$
\begin{aligned}
\alpha^{P I} & =\frac{N(N-1) \sigma_{v}^{2}}{\left(N \sigma_{v}^{2}+\sigma_{e}^{2}\right)} \gamma^{P I}\left(\frac{\sigma_{e}^{2}\left(N(3 N-2) \sigma_{v}^{2}+(2 N-1)\right)}{N(N-1) \sigma_{v}^{2}\left(N^{2} \sigma_{v}^{2}+\sigma_{e}^{2}(2 N-1)\right)} \bar{v}+\frac{A}{N} \bar{m}\right) \\
& =\frac{\gamma^{P I}}{\left(N \sigma_{v}^{2}+\sigma_{e}^{2}\right)} \frac{\sigma_{e}^{2}\left(N(3 N-2) \sigma_{v}^{2}+(2 N-1)\right)}{\left(N^{2} \sigma_{v}^{2}+\sigma_{e}^{2}(2 N-1)\right)} \bar{v}+\frac{(N-1) \sigma_{v}^{2}}{\left(N \sigma_{v}^{2}+\sigma_{e}^{2}\right)} \gamma^{P I} A \bar{m} \\
& =\frac{\sigma_{e}^{2}\left(N(3 N-2) \sigma_{v}^{2}+(2 N-1)\right)}{2 N^{2} \sigma_{v}^{2}\left(N^{2} \sigma_{v}^{2}+\sigma_{e}^{2}\right)\left(N \sigma_{v}^{2}+\sigma_{e}^{2}\right)} \sqrt{\delta} \bar{v}+\frac{N(N-2) \sigma_{v}^{2}-\sigma_{e}^{2}}{N\left(N^{2} \sigma_{v}^{2}+\sigma_{e}^{2}\right)} \bar{m}
\end{aligned}
$$

Here we have defined $\delta$ by

$$
\delta \equiv \frac{\left(N(N-2) \sigma_{v}^{2}-\sigma_{e}^{2}\right)\left(N^{2} \sigma_{v}^{2}+\sigma_{e}^{2}\right) \sigma_{S}^{2}}{(N-1) \sigma_{e}^{2}}
$$

Similarly,

$$
\begin{aligned}
\alpha^{S I}= & N \gamma^{P I}\left(\bar{v}\left(1-C N \beta^{P I}\right)-C t\right)=\left(-\frac{(N-1) \sigma_{e}^{2} \gamma^{P I}}{\left(N \sigma_{v}^{2}+\sigma_{e}^{2}\right)}\right) \bar{v}+C \gamma^{P I} \bar{m} \\
& =\left(-\frac{(N-1) \sigma_{e}^{2}}{\left(N \sigma_{v}^{2}+\sigma_{e}^{2}\right)} \frac{N^{2} \sigma_{v}^{2}+(2 N-1) \sigma_{e}^{2}}{2 N^{2} \sigma_{v}^{2}\left(N^{2} \sigma_{v}^{2}+\sigma_{e}^{2}\right)} \sqrt{\delta}\right) \bar{v}+\frac{N^{2} \sigma_{v}^{2}+(2 N-1) \sigma_{e}^{2}}{N\left(N^{2} \sigma_{v}^{2}+\sigma_{e}^{2}\right)} \bar{m} .
\end{aligned}
$$

Consequently, using the definition of $\delta$ we can write the coefficients in the following 
way:

$$
\begin{aligned}
\alpha^{P I} & =\frac{\sigma_{e}^{2}\left(N(3 N-2) \sigma_{v}^{2}+(2 N-1) \sigma_{e}^{2}\right) \delta^{1 / 2}}{2 N^{2} \sigma_{v}^{2}\left(N^{2} \sigma_{v}^{2}+\sigma_{e}^{2}\right)\left(N \sigma_{v}^{2}+\sigma_{e}^{2}\right)} \bar{v}+\frac{N(N-2) \sigma_{v}^{2}-\sigma_{e}^{2}}{N\left(N^{2} \sigma_{v}^{2}+\sigma_{e}^{2}\right)} \bar{m} \\
\beta^{P I} & =\frac{\delta^{1 / 2}}{2 N\left(N \sigma_{v}^{2}+\sigma_{e}^{2}\right)} \\
\gamma^{P I} & =\frac{\left(N^{2} \sigma_{v}^{2}+(2 N-1) \sigma_{e}^{2}\right) \delta^{1 / 2}}{2 N^{2} \sigma_{v}^{2}\left(N^{2} \sigma_{v}^{2}+\sigma_{e}^{2}\right)} \\
\alpha^{S I} & =\left(-\frac{(N-1) \sigma_{e}^{2}}{\left(N \sigma_{v}^{2}+\sigma_{e}^{2}\right)} \frac{N^{2} \sigma_{v}^{2}+(2 N-1) \sigma_{e}^{2}}{2 N^{2} \sigma_{v}^{2}\left(N^{2} \sigma_{v}^{2}+\sigma_{e}^{2}\right)} \delta^{1 / 2}\right) \bar{v}+\frac{N^{2} \sigma_{v}^{2}+(2 N-1) \sigma_{e}^{2}}{N\left(N^{2} \sigma_{v}^{2}+\sigma_{e}^{2}\right)} \bar{m} \\
\beta^{S I} & =\frac{N^{2} \sigma_{v}^{2}+(2 N-1) \sigma_{e}^{2}}{2 N\left(N \sigma_{v}^{2}+\sigma_{e}^{2}\right)} \\
\gamma^{S I} & =-\frac{(N-1) \sigma_{e}^{2} N^{2} \sigma_{v}^{2}+(2 N-1) \sigma_{e}^{2}}{\left(N \sigma_{v}^{2}+\sigma_{e}^{2}\right)} \frac{N^{2} \sigma_{v}^{2}\left(N^{2} \sigma_{v}^{2}+\sigma_{e}^{2}\right)}{\delta^{1 / 2}}
\end{aligned}
$$

Proof of Corollary 1. While solving the above system we have obtained that

$$
\gamma=N \gamma^{P I}+\gamma^{S I}=\frac{N^{2} \sigma_{v}^{2}+(2 N-1) \sigma_{e}^{2}}{2 N^{2} \sigma_{v}^{2}\left(N \sigma_{v}^{2}+\sigma_{e}^{2}\right)}\left(\frac{\left(N(N-2) \sigma_{v}^{2}-\sigma_{e}^{2}\right)\left(N^{2} \sigma_{v}^{2}+\sigma_{e}^{2}\right) \sigma_{S}^{2}}{(N-1) \sigma_{e}^{2}}\right)^{1 / 2}
$$

We study first how market depth varies when the variance of liquidity shock $\widetilde{S}$ varies. We compute the derivative $\frac{\partial \gamma}{\partial \sigma_{S}^{2}}$ and we obtain

$$
\frac{\partial \gamma}{\partial \sigma_{S}^{2}}>0
$$

Then we calculate $\frac{\partial \gamma}{\partial \sigma_{e}^{2}}$ and after somehow tedious calculations we obtain that

$$
\frac{\partial \gamma}{\partial \sigma_{e}^{2}}<0
$$

Finally, we study how the variance of liquidation value, $\sigma_{v}^{2}$ affects the market depth. We calculate the derivative $\frac{\partial \gamma}{\partial \sigma_{v}^{2}}$ and we obtain that this expression has the opposite sign to $f\left(\sigma_{v}^{2}\right)$, where

$$
\begin{aligned}
f\left(\sigma_{v}^{2}\right)= & N^{4}\left(\sigma_{v}^{2}\right)^{3}(N-1)\left(N^{2}-3 N+1\right)-3 \sigma_{e}^{2} N^{2}\left(\sigma_{v}^{2}\right)^{2}(2 N-1)(N-1) \\
& -3 \sigma_{v}^{2}\left(\sigma_{e}^{2}\right)^{2} N(2 N-1)(N-1)-\left(\sigma_{e}^{2}\right)^{3}(2 N-1)(N-1) .
\end{aligned}
$$


We study this function and we obtain that the equation $f^{\prime}\left(\sigma_{v}^{2}\right)=0$,

$f^{\prime}\left(\sigma_{v}^{2}\right)=3(N-1) N\left[\left(N^{3}\left(\sigma_{v}^{2}\right)^{2}\left(N^{2}-3 N+1\right)-2 \sigma_{e}^{2} N(2 N-1) \sigma_{v}^{2}-\left(\sigma_{e}^{2}\right)^{2}(2 N-1)\right)\right]$,

has only one positive solution equal to

$$
\sigma_{e}^{2} \frac{(2 N-1)+(N-1)((2 N-1)(N-1))^{1 / 2}}{N^{2}\left(N^{2}-3 N+1\right)} \equiv k_{l}(N) .
$$

We obtain that $k_{l}(N)>\frac{1}{N(N-2)}$. So, it results that the function $f\left(\sigma_{v}^{2}\right)$ is decreasing for $\frac{\sigma_{v}^{2}}{\sigma_{e}^{2}} \in\left[\frac{1}{N(N-2)}, k_{l}(N)\right]$, and is increasing for $\frac{\sigma_{v}^{2}}{\sigma_{e}^{2}}>k_{l}(N)$. Since $f(0)=$ $-\left(\sigma_{e}^{2}\right)^{3}(2 N-1)(N-1)$, it results that it exists $k^{*}\left(N, \sigma_{e}^{2}\right)$ such that $f\left(k^{*}\left(N, \sigma_{e}^{2}\right)\right)$ $=0$. Therefore, the function $f\left(\sigma_{v}^{2}\right)<0$ for any $\sigma_{v}^{2}<k^{*}\left(N, \sigma_{e}^{2}\right)$ and is greater than 0 otherwise.

Once we have characterized the behavior of function $f\left(\sigma_{v}^{2}\right)$ we can conclude that the market depth is a increasing function of $\sigma_{v}^{2}$ if $\sigma_{v}^{2}<k^{*}\left(N, \sigma_{e}^{2}\right)$ and is decreasing otherwise.

Proof of Corollary 2. From the market clearing condition (6) we obtain that the equilibrium price is

$$
\widetilde{p}=\left(N \gamma^{P I}+\gamma^{S I}\right)^{-1}\left(\alpha+\beta^{P I} \sum_{n=1}^{N} \widetilde{i_{n}}-\left(1-\beta^{S I}\right) \widetilde{S}-\bar{m}\right) .
$$

We had obtained in the proof of Proposition 1 that

$$
\begin{gathered}
\alpha=\frac{\sigma_{e}^{2}(2 N-1)}{2 N^{2} \sigma_{v}^{2}\left(N \sigma_{v}^{2}+\sigma_{e}^{2}\right)} \delta^{1 / 2} \bar{v}+\frac{(N-1)}{N} \bar{m} \\
N \gamma^{P I}+\gamma^{S I}=\frac{\left(N^{2} \sigma_{v}^{2}+(2 N-1) \sigma_{e}^{2}\right) \delta^{1 / 2}}{2 N^{2} \sigma_{v}^{2}\left(N \sigma_{v}^{2}+\sigma_{e}^{2}\right)} .
\end{gathered}
$$

Using these formulas and the ones for $\beta^{P I}$ and $\beta^{S I}$ we can write that the equilibrium price equals to

$$
\begin{aligned}
\widetilde{p}= & \frac{\sigma_{e}^{2}(2 N-1)}{N^{2} \sigma_{v}^{2}+(2 N-1) \sigma_{e}^{2}} \bar{v}+\frac{N \sigma_{v}^{2}}{N^{2} \sigma_{v}^{2}+(2 N-1) \sigma_{e}^{2}} \sum_{n=1}^{N} \widetilde{i_{n}} \\
& -\frac{N \sigma_{v}^{2}\left(N^{2} \sigma_{v}^{2}+\sigma_{e}^{2}\right)}{\left(N^{2} \sigma_{v}^{2}+(2 N-1) \sigma_{e}^{2}\right) \delta^{1 / 2}} \widetilde{S}-\frac{2 N \sigma_{v}^{2}\left(N \sigma_{v}^{2}+\sigma_{e}^{2}\right)}{\left(N^{2} \sigma_{v}^{2}+(2 N-1) \sigma_{e}^{2}\right) \delta^{1 / 2}} \bar{m} .
\end{aligned}
$$


Notice that since $\widetilde{i_{n}}=\widetilde{v}+\widetilde{e_{n}}$ we can write

$$
\begin{gathered}
\widetilde{p}=\frac{\sigma_{e}^{2}(2 N-1)}{N^{2} \sigma_{v}^{2}+(2 N-1) \sigma_{e}^{2}} \bar{v}+\frac{N^{2} \sigma_{v}^{2}}{N^{2} \sigma_{v}^{2}+(2 N-1) \sigma_{e}^{2}} \widetilde{v}+\frac{N \sigma_{v}^{2}}{N^{2} \sigma_{v}^{2}+(2 N-1) \sigma_{e}^{2}} \sum_{n=1}^{N} \widetilde{e_{n}} \\
-\frac{N \sigma_{v}^{2}\left(N^{2} \sigma_{v}^{2}+\sigma_{e}^{2}\right)}{\left(N^{2} \sigma_{v}^{2}+(2 N-1) \sigma_{e}^{2}\right) \delta^{1 / 2}} \widetilde{S}-\frac{2 N \sigma_{v}^{2}\left(N \sigma_{v}^{2}+\sigma_{e}^{2}\right)}{\left(N^{2} \sigma_{v}^{2}+(2 N-1) \sigma_{e}^{2}\right) \delta^{1 / 2}} \bar{m} .
\end{gathered}
$$

Taking the expectations it results that $E(\widetilde{p})=\bar{v}-\frac{2 N \sigma_{v}^{2}\left(N \sigma_{v}^{2}+\sigma_{e}^{2}\right)}{\left(N^{2} \sigma_{v}^{2}+(2 N-1) \sigma_{e}^{2}\right) \delta^{1 / 2}} \bar{m}$

Proof of Corollary 3. We have seen that the equilibrium price is given by (5). As a result, we can compute the variance

$$
\begin{aligned}
\operatorname{Var}(\widetilde{p})= & \operatorname{Var}\left(\frac{\sigma_{e}^{2}(2 N-1)}{N^{2} \sigma_{v}^{2}+(2 N-1) \sigma_{e}^{2}} \bar{v}+\frac{N \sigma_{v}^{2}}{N^{2} \sigma_{v}^{2}+(2 N-1) \sigma_{e}^{2}} \sum_{n=1}^{N} \widetilde{i_{n}}\right. \\
& \left.-\frac{N \sigma_{v}^{2}\left(N^{2} \sigma_{v}^{2}+\sigma_{e}^{2}\right)}{\left(N^{2} \sigma_{v}^{2}+(2 N-1) \sigma_{e}^{2}\right) \delta^{1 / 2}} \widetilde{S}-\frac{2 N \sigma_{v}^{2}\left(N \sigma_{v}^{2}+\sigma_{e}^{2}\right)}{\left(N^{2} \sigma_{v}^{2}+(2 N-1) \sigma_{e}^{2}\right) \delta^{1 / 2}} \bar{m}\right)= \\
& \frac{N^{2}(N-2)\left(\sigma_{v}^{2}\right)^{2}+N \sigma_{v}^{2} \sigma_{e}^{2}\left(2 N^{2}-3 N-1\right)-\left(\sigma_{e}^{2}\right)^{2}}{\left(N(N-2) \sigma_{v}^{2}-\sigma_{e}^{2}\right)}\left(\frac{N \sigma_{v}^{2}}{N^{2} \sigma_{v}^{2}+(2 N-1) \sigma_{e}^{2}}\right)^{2} .
\end{aligned}
$$

Proof of Corollary 4. We compute now $\operatorname{Var}(\widetilde{v})-\operatorname{Var}(\widetilde{v} \mid \widetilde{p})$. Due to the normality assumptions we have that

$$
\operatorname{Var}(\widetilde{v})-\operatorname{Var}(\widetilde{v} \mid \widetilde{p})=(\operatorname{Var}(\widetilde{p}))^{-1}(\operatorname{Cov}(\widetilde{v}, \widetilde{p}))^{2}
$$

We calculate the covariance

$$
\operatorname{Cov}(\widetilde{v}, \widetilde{p})=\frac{\left(N \sigma_{v}^{2}\right)^{2}}{N^{2} \sigma_{v}^{2}+(2 N-1) \sigma_{e}^{2}}
$$

and together with the formula for variance $\operatorname{Var}(\widetilde{p})$ we obtained before, we plug them above to obtain

$$
\operatorname{Var}(\widetilde{v})-\operatorname{Var}(\widetilde{v} \mid \widetilde{p})=\frac{N \sigma_{v}^{2}\left(N(N-2) \sigma_{v}^{2}-\sigma_{e}^{2}\right)}{N^{2}(N-2)\left(\sigma_{v}^{2}\right)^{2}+N \sigma_{v}^{2} \sigma_{e}^{2}\left(2 N^{2}-3 N-1\right)-\left(\sigma_{e}^{2}\right)^{2}} .
$$

Proof of Corollary 5. Since the demand of the price informed agent $x_{n}$ can be written as the sum of normal variables it results that $x_{n}$ is also a normal variable. The 
mean of $x_{n}$ is $\mu_{n}=\frac{(N-1) \sigma_{v}^{2}}{\left(N^{2} \sigma_{v}^{2}+\sigma_{e}^{2}\right)} \bar{m}$ while the variance $\sigma_{x_{\mathrm{n}}}$ is

$$
\begin{aligned}
\sigma_{x_{\mathrm{n}}}=\operatorname{Var}\left(x_{n}\right) & =\left(\frac{(N-1) \sigma_{e}^{2} \delta^{1 / 2}}{2 N\left(N^{2} \sigma_{v}^{2}+\sigma_{e}^{2}\right)\left(N \sigma_{v}^{2}+\sigma_{e}^{2}\right)} \bar{v}+\frac{(N-1) \sigma_{v}^{2}}{\left(N^{2} \sigma_{v}^{2}+\sigma_{e}^{2}\right)} \bar{m}\right. \\
+ & \left.\frac{\delta^{1 / 2}}{2 N\left(N \sigma_{v}^{2}+\sigma_{e}^{2}\right)} \tilde{i_{n}}-\frac{\delta^{1 / 2}}{2 N\left(N^{2} \sigma_{v}^{2}+\sigma_{e}^{2}\right)} \sum_{n=1}^{N} \tilde{i_{n}}+\frac{1}{2 N} \widetilde{S}\right)= \\
& \left(\frac{\delta^{1 / 2}}{2 N\left(N \sigma_{v}^{2}+\sigma_{e}^{2}\right)}\right)^{2}\left(\sigma_{v}^{2}+\sigma_{e}^{2}\right)+\left(\frac{\delta^{1 / 2}}{2 N\left(N^{2} \sigma_{v}^{2}+\sigma_{e}^{2}\right)}\right)^{2} N\left(\sigma_{v}^{2}+\sigma_{e}^{2}\right) \\
& +\frac{1}{4 N^{2}} \sigma_{S}^{2}=\frac{\left(\sigma_{v}^{2}+\sigma_{e}^{2}\right) \delta}{4 N^{2}}\left(\frac{1}{\left(N \sigma_{v}^{2}+\sigma_{e}^{2}\right)^{2}}+\frac{N}{\left(N^{2} \sigma_{v}^{2}+\sigma_{e}^{2}\right)^{2}}\right)+\frac{\sigma_{S}^{2}}{4 N^{2}} .
\end{aligned}
$$

Then, since $x_{n}$ is $\mathcal{N}\left(\mu_{n}, \sigma_{x_{\mathrm{n}}}\right)$ it results that the expected volume of trade

$$
\begin{aligned}
& E\left(\left|x_{n}\right|\right)=\int_{-\infty}^{\infty}\left|x_{n}\right| \frac{1}{\sigma_{x_{\mathrm{n}}} \sqrt{2 \pi}} \exp \left(-\frac{\left(x_{n}-\mu_{n}\right)^{2}}{2 \sigma_{x_{\mathrm{n}}}^{2}}\right) d x_{n}=2 \mu_{n}+\left(\frac{2}{\pi}\right)^{2} \sigma_{x_{\mathrm{n}}}= \\
& \frac{2(N-1) \sigma_{v}^{2}}{\left(N^{2} \sigma_{v}^{2}+\sigma_{e}^{2}\right)} \bar{m}+\left(\frac{2}{\pi}\right)^{2}\left(\frac{\left(\sigma_{v}^{2}+\sigma_{e}^{2}\right) \delta}{4 N^{2}}\left(\frac{1}{\left(N \sigma_{v}^{2}+\sigma_{e}^{2}\right)^{2}}+\frac{N}{\left(N^{2} \sigma_{v}^{2}+\sigma_{e}^{2}\right)^{2}}\right)+\frac{1}{4 N^{2}} \sigma_{S}^{2}\right) .
\end{aligned}
$$

Similarly, the quantity demanded by the supply informed agent is a normal variable with mean $\mu_{y}=\frac{\left(N \sigma_{v}^{2}+\sigma_{e}^{2}\right)}{\left(N^{2} \sigma_{v}^{2}+\sigma_{e}^{2}\right)} \bar{m}$ and variance

$$
\begin{aligned}
\sigma_{y} & =\operatorname{Var}(y)=\left(\frac{(N-1) \sigma_{e}^{2} \delta^{1 / 2}}{2 N\left(N^{2} \sigma_{v}^{2}+\sigma_{e}^{2}\right)\left(N \sigma_{v}^{2}+\sigma_{e}^{2}\right)}\right)^{2} N\left(\sigma_{v}^{2}+\sigma_{e}^{2}\right)+\frac{1}{4} \sigma_{S}^{2} \\
& =\frac{1}{4} \sigma_{S}^{2}\left(1+\frac{(N-1) \sigma_{e}^{2}\left(N(N-2) \sigma_{v}^{2}-\sigma_{e}^{2}\right)\left(\sigma_{v}^{2}+\sigma_{e}^{2}\right)}{N\left(N^{2} \sigma_{v}^{2}+\sigma_{e}^{2}\right)\left(N \sigma_{v}^{2}+\sigma_{e}^{2}\right)^{2}}\right) .
\end{aligned}
$$

Then since $y$ is $\mathcal{N}\left(\mu_{y}, \sigma_{y}\right)$ it results that the expected volume of trade of the supply informed agent is

$$
\begin{aligned}
& E(|y|)=\int_{-\infty}^{\infty}|y| \frac{1}{\sigma_{y} \sqrt{2 \pi}} \exp \left(-\frac{\left(y-\mu_{y}\right)^{2}}{2 \sigma_{y}^{2}}\right) d y=2 \mu_{y}+\sqrt{\frac{2}{\pi}} \sigma_{y} \\
= & 2 \frac{\left(N \sigma_{v}^{2}+\sigma_{e}^{2}\right)}{\left(N^{2} \sigma_{v}^{2}+\sigma_{e}^{2}\right)} \bar{m}+\left(\frac{2}{\pi}\right)^{1 / 2} \frac{1}{4} \sigma_{S}^{2}\left(1+\frac{(N-1) \sigma_{e}^{2}\left(N(N-2) \sigma_{v}^{2}-\sigma_{e}^{2}\right)\left(\sigma_{v}^{2}+\sigma_{e}^{2}\right)}{N\left(N^{2} \sigma_{v}^{2}+\sigma_{e}^{2}\right)\left(N \sigma_{v}^{2}+\sigma_{e}^{2}\right)^{2}}\right) .
\end{aligned}
$$


Proof of Corollary 6. Let us compute first the unconditional expected profit of the $n^{\text {th }}$ price informed trader.

$$
\Pi_{n}^{P I}=E\left(\widetilde{\pi_{n}^{P I}}\right)=E\left((\widetilde{v}-\widetilde{p}) \widetilde{x_{n}}\right) .
$$

Using the formulas we have obtained for $\widetilde{p}$ and $\widetilde{x_{n}}$ we can write further

$$
\begin{aligned}
& \Pi_{n}^{P I}=E\left(\frac{\sigma_{e}^{2}(2 N-1)}{N^{2} \sigma_{v}^{2}+(2 N-1) \sigma_{e}^{2}}(\widetilde{v}-\bar{v})-\frac{N \sigma_{v}^{2}}{N^{2} \sigma_{v}^{2}+(2 N-1) \sigma_{e}^{2}} \sum_{n=1}^{N} \widetilde{e_{n}}+\right. \\
&\left.\frac{N \sigma_{v}^{2}\left(N^{2} \sigma_{v}^{2}+\sigma_{e}^{2}\right)}{\left(N^{2} \sigma_{v}^{2}+(2 N-1) \sigma_{e}^{2}\right) \delta^{1 / 2}} \widetilde{S}+\frac{2 N \sigma_{v}^{2}\left(N \sigma_{v}^{2}+\sigma_{e}^{2}\right)}{\left(N^{2} \sigma_{v}^{2}+(2 N-1) \sigma_{e}^{2}\right) \delta^{1 / 2}} \bar{m}\right) \\
&\left(-\frac{\delta^{1 / 2}(N-1) \sigma_{e}^{2}}{2 N\left(N^{2} \sigma_{v}^{2}+\sigma_{e}^{2}\right)\left(N \sigma_{v}^{2}+\sigma_{e}^{2}\right)}(\widetilde{v}-\bar{v})+\frac{\delta^{1 / 2}}{2 N\left(N \sigma_{v}^{2}+\sigma_{e}^{2}\right)} \widetilde{e_{n}}\right. \\
&\left.-\frac{1}{2 N\left(N^{2} \sigma_{v}^{2}+\sigma_{e}^{2}\right)} \delta^{1 / 2} \sum_{k=1}^{N} \widetilde{e_{k}}+\frac{1}{2 N} \widetilde{S}+\frac{(N-1) \sigma_{v}^{2}}{\left(N^{2} \sigma_{v}^{2}+\sigma_{e}^{2}\right)} \bar{m}\right)= \\
& \frac{\sigma_{v}^{2} \delta^{1 / 2}(N-1) \sigma_{e}^{2}}{2 N\left(N^{2} \sigma_{v}^{2}+(2 N-1) \sigma_{e}^{2}\right)\left(N \sigma_{v}^{2}+\sigma_{e}^{2}\right)}\left(\frac{N\left(N \sigma_{v}^{2}+\sigma_{e}^{2}\right)}{\left(N(N-2) \sigma_{v}^{2}-\sigma_{e}^{2}\right)}-\frac{(N-1) \sigma_{e}^{2}}{\left(N^{2} \sigma_{v}^{2}+\sigma_{e}^{2}\right)}\right)+ \\
&+\frac{(N-1) \sigma_{v}^{2}}{\left(N^{2} \sigma_{v}^{2}+\sigma_{e}^{2}\right)} \frac{2 N \sigma_{v}^{2}\left(N \sigma_{v}^{2}+\sigma_{e}^{2}\right)}{\left(N^{2} \sigma_{v}^{2}+(2 N-1) \sigma_{e}^{2}\right) \delta^{1 / 2}} \bar{m}^{2} .
\end{aligned}
$$

Let us compute now the unconditional expected profit of the supply informed trader. Using the formulas we have obtained for $\widetilde{p}$ and $\widetilde{y}$ we can write further

$$
\begin{gathered}
\Pi^{S I}=E\left(\widetilde{\pi^{S I}}\right)=E((\widetilde{v}-\widetilde{p}) \widetilde{y}) . \\
\Pi^{S I}=E\left(\left(\frac{\sigma_{e}^{2}(2 N-1)}{N^{2} \sigma_{v}^{2}+(2 N-1) \sigma_{e}^{2}}(\widetilde{v}-\bar{v})-\frac{N \sigma_{v}^{2}}{N^{2} \sigma_{v}^{2}+(2 N-1) \sigma_{e}^{2}} \sum_{n=1}^{N} \widetilde{e_{n}}+\right.\right. \\
\left.\frac{N \sigma_{v}^{2}\left(N^{2} \sigma_{v}^{2}+\sigma_{e}^{2}\right)}{\left(N^{2} \sigma_{v}^{2}+(2 N-1) \sigma_{e}^{2}\right) \delta^{1 / 2}} \widetilde{S}+\frac{2 N \sigma_{v}^{2}\left(N \sigma_{v}^{2}+\sigma_{e}^{2}\right)}{\left(N^{2} \sigma_{v}^{2}+(2 N-1) \sigma_{e}^{2}\right) \delta^{1 / 2}} \bar{m}\right) \\
\left(\frac{(N-1) \sigma_{e}^{2} \delta^{1 / 2}}{2\left(N^{2} \sigma_{v}^{2}+\sigma_{e}^{2}\right)\left(N \sigma_{v}^{2}+\sigma_{e}^{2}\right)}(\widetilde{v}-\bar{v})+\frac{\left(N \sigma_{v}^{2}+\sigma_{e}^{2}\right)}{\left(N^{2} \sigma_{v}^{2}+\sigma_{e}^{2}\right)} \bar{m}\right. \\
\left.+\frac{(N-1) \sigma_{e}^{2} \delta^{1 / 2}}{2 N\left(N^{2} \sigma_{v}^{2}+\sigma_{e}^{2}\right)\left(N \sigma_{v}^{2}+\sigma_{e}^{2}\right)} \sum_{n=1}^{N} \widetilde{e_{n}}+\frac{1}{2} \widetilde{S}\right)= \\
\frac{(N-1) \sigma_{e}^{2}}{2\left(N^{2} \sigma_{v}^{2}+(2 N-1) \sigma_{e}^{2}\right)}\left(\frac{N}{\left(N^{2} \sigma_{v}^{2}+\sigma_{e}^{2}\right)\left(N \sigma_{v}^{2}+\sigma_{e}^{2}\right)}+\frac{2 N \sigma_{v}^{2}\left(N \sigma_{v}^{2}+\sigma_{e}^{2}\right)}{\left.(N-2) \sigma_{v}^{2}-\sigma_{e}^{2}\right)}\right) \\
+\frac{\left(N \sigma_{v}^{2}+\sigma_{e}^{2}\right)}{\left(N^{2} \sigma_{v}^{2}+(2 N-1) \sigma_{e}^{2}\right) \delta^{1 / 2}} \frac{\left(N^{2} \sigma_{v}^{2}+\sigma_{e}^{2}\right)}{\delta^{2}} .
\end{gathered}
$$


The total profits in the market are

$$
\Pi=N \Pi^{P I}+\Pi^{S I}=E\left((\widetilde{v}-\widetilde{p})\left(\sum_{n=1}^{N} \widetilde{x_{n}}+\widetilde{y}\right)\right) .
$$

But from the market clearing condition it results that

$$
\begin{aligned}
\Pi= & N \Pi^{P I}+\Pi^{S I}=E((\widetilde{v}-\widetilde{p})(\bar{m}+\widetilde{S}))= \\
& E\left(\frac{\sigma_{e}^{2}(2 N-1)}{N^{2} \sigma_{v}^{2}+(2 N-1) \sigma_{e}^{2}}(\widetilde{v}-\bar{v})-\frac{N \sigma_{v}^{2}}{N^{2} \sigma_{v}^{2}+(2 N-1) \sigma_{e}^{2}} \sum_{n=1}^{N} \widetilde{e_{n}}\right. \\
& \left.+\frac{N \sigma_{v}^{2}\left(N^{2} \sigma_{v}^{2}+\sigma_{e}^{2}\right)}{\left(N^{2} \sigma_{v}^{2}+(2 N-1) \sigma_{e}^{2}\right) \delta^{1 / 2}} \widetilde{S}+\frac{2 N \sigma_{v}^{2}\left(N \sigma_{v}^{2}+\sigma_{e}^{2}\right)}{\left(N^{2} \sigma_{v}^{2}+(2 N-1) \sigma_{e}^{2}\right) \delta^{1 / 2}} \bar{m}\right)(\bar{m}+\widetilde{S}) \\
= & \frac{N \sigma_{v}^{2}}{\left(N^{2} \sigma_{v}^{2}+(2 N-1) \sigma_{e}^{2}\right) \delta^{1 / 2}}\left(\left(N^{2} \sigma_{v}^{2}+\sigma_{e}^{2}\right) \sigma_{S}^{2}+2\left(N \sigma_{v}^{2}+\sigma_{e}^{2}\right) \bar{m}^{2}\right) .
\end{aligned}
$$

We can check and see that indeed the profits we have obtained sum up to this amount.

\section{R eferences}

[1] Admati, A. and P. Pfleiderer, 1991, Sunshine Trading and Financial Market Equilibrium, Review of Financial Studies, 4(3), 443-482.

[2] Bhattacharya, U. and M. Spiegel, 1991, Insiders, Outsiders, and Market Breakdowns, Review of Financial Studies 4, 255-282.

[3] Brown, D. and Z.P. Zhang, 1997, Markets Orders and Market Efficiency, Journal of Finance 52, 277-308.

[4] Caballé, J., 1992, Market Versus Limit Orders, Economic Letters, 40, 339-344.

[5] Caballé, J. and M. Krishnan, 1994, Imperfect Competition in a Multi-Security Market with Risk Neutrality, Econometrica 62, 695-704.

[6] Ellis,K., Michaely, R. and M. O'Hara, 2001, The Making of a Deal Market: From Entry to Equilibrium in the Trading of Nasdaq Stocks, Working paper, Cornell University. 
[7] Foster, F.D. and S. Wiswanathan, 1993, Variations in Trading Volume, Return Volatility, and Trading Costs: Evidence on Recent Price Formation Models, Journal of Finance, 48, 187-211.

[8] Froot, K., Scharfstein, D. and J. Stein, 1992, Herd on the Street: Informational Inefficiencies in a Market with Short-Term Speculation, The Journal of Finance, 47, 1461-1484.

[9] Gennotte, G. and H. Leland, 1990, Market Liquidity, Hedging and Crashes, American Economic Review 80, 999-1021.

[10] Glosten, L. and P. Milgrom, 1985, Bid, Ask and Transaction Prices in a Specialist Market with Heterogeneously Informed Markets, Journal of Financial Economics, 14, 71-100.

[11] Grossman, S. and S. Stiglitz, 1980, On the Impossibility of Informationally Efficient Markets, American Economic Review 70, 393-408.

[12] Holden, C.W. and A. Subrahmanyam, 1992, Long-Lived Private Information and Imperfect Competition, Journal of Finance, 47, 247-270.

[13] Jackson, M., 1991, Equilibrium, Price Formation and the Value of Private Information, Review of Financial Studies 4, 1-16.

[14] Kyle, A.S., 1985, Continuous Auctions and Insider Trading, Econometrica, 53, 1315-1336.

[15] Kyle, A.S., 1989, Informed Speculators with Imperfect Competition, Review of Economic Studies 56, 317-356.

[16] O'Hara, M., 1995, Market Microstructure Theory, Blackwell Publishers, Cambridge, Massachusetts.

[17] Madhavan, A., 1992, Trading Mechanism in Security Markets, Journal of Finance, 47(2), 607-642. 
[18] Palomino, F., 2001, Informational Efficiency: Ranking Markets, Economic Theory, 18(3), 683-700.

[19] Rochet, J.C. and J.L. Vila, 1994, Insider Trading without Normality, Review of Economic Studies, 61, 131-152.

[20] Subrahmanyam, A., 1991, Risk Aversion, Market Liquidity, and Price Efficiency, Review of Financial Studies, 4(3), 417-441. 